\title{
Exact solution for the unforced Duffing oscillator with cubic and quintic nonlinearities
}

\author{
A. Beléndez ${ }^{1,2}$, T. Beléndez ${ }^{1,2}$, F. J. Martínez $^{2}$, C. Pascual ${ }^{1,2}$, M. L. \\ Alvarez $^{1,2}$ and E. Arribas ${ }^{3}$
}

(1) Departamento de Física, Ingeniería de Sistemas y Teoría de la Señal.

Universidad de Alicante. Apartado 99. E-03080 Alicante. SPAIN

(2) Instituto Universitario de Física Aplicada a las Ciencias y las Tecnologías.

Universidad de Alicante. Apartado 99. E-03080 Alicante. SPAIN

(3) Departamento de Física Aplicada. Universidad de Castilla-La Mancha.

Avda. España s/n, E-02071 Albacete. SPAIN

\begin{abstract}
The nonlinear differential equation governing the periodic motion of the one-dimensional, undamped, unforced cubic-quintic Duffing oscillator is solved exactly, providing exact expressions for the period and the solution. The period is given in terms of the complete elliptic integral of the first kind and the solution involves Jacobi elliptic functions. Some particular cases obtained varying the parameters that characterize this oscillator are presented and discussed. The behaviour of the period as a function of the initial amplitude is analysed, the exact solutions and velocities for several values of the initial amplitude are plotted and the Fourier series expansions for the exact solutions are also obtained. All this allows us to conclude that the quintic term appearing in the cubic-quintic Duffing equation makes this nonlinear oscillator not only more complex but also more interesting to study.
\end{abstract}

Keywords: Dynamical systems; Nonlinear oscillators; Conservative systems; Cubic-quintic nonlinear oscillator; Exact solution; Jacobian elliptic functions; Symbolic computation. 


\section{Introduction}

Nonlinear oscillators in physics, engineering, mathematical and related fields have been the focus of attention for many years and several methods have been used to find approximate solutions to these dynamical systems [1,2]. In conservative nonlinear oscillators the restoring force is not dependent on time, the total energy is constant $[2,3]$ and any oscillation is stationary. An important feature of the solutions of conservative oscillators is that they are periodic and range over a continuous interval of initial values [2]. The cubic-quintic nonlinear oscillator is described by a differential equation with third- and fifth-power nonlinearity. As Lai et al. pointed out [4], the cubic-quintic Duffing equation can be found in the modelling of different systems and we advise readers to consult this paper to learn more about these applications and obtain a good set of references in which this strongly nonlinear equation can be found. The systems modelled by the cubic-quintic Duffing equation include the nonlinear dynamics of a slender elastica, the compound $\mathrm{KdV}$ equation in nonlinear wave systems, or the propagation of a short electromagnetic pulse in a nonlinear medium [4-6]. Due to the presence of the fifth-power nonlinearity added to the third nonlinearity of the common Duffing equation, this oscillator is difficult to handle and has not been studied as extensively as the Duffing oscillator with cubic nonlinearity. For this reason, several techniques have been used to obtain analytical approximate expressions for the period and the solution of the cubic-quintic Duffing oscillator.

Lin [7] proposed a new parameter iteration technique to solve the Duffing equation with strong and higher order nonlinearity. Ramos [6] approximately solved the quintic Duffing equation using some Linstedt-Poincaré techniques and Pirbodaghi et al. [8] obtained an accurate analytical approximate solution to Duffing equations with cubic and quintic nonlinearities using the homotopy analysis method and homotopy Padé technique. Wu et al. [9] approximately solved this nonlinear oscillator using a method that incorporates salient features of both Newton's method and the harmonic balance method. Later, Lai et al. [4] used a Newton-harmonic balancing approach to obtain accurate approximate analytical higher-order solutions for strong nonlinear Duffing oscillators with cubic-quintic restoring force. They also discussed the effect of strong quintic nonlinearity on accuracy as compared to cubic nonlinearity. Beléndez et al. $[10,11]$ approximately solved the quintic and the cubicquintic Duffing oscillators using a cubication method which allowed them to obtain approximate analytical expressions for the period and the solution in terms of elementary functions. Scarpello [12] exactly solved the quintic Duffing oscillator. He obtained the 
closed form relationship between the period and the initial motion amplitude as well as the exact expression for the solution which is written in terms of the cs Jacobian elliptic function and the complete elliptic integral of the first kind. Chua [13] investigated unforced and forced cubic-quintic Duffing oscillators, finding their equilibrium points and employing perturbative analytical techniques. In particular Chua derived approximate periodic solutions and period-amplitude relations. Elías-Zúñiga [14] derived the exact solution of the cubicquintic Duffing equation based on the use of Jacobi elliptic functions. However, he does not solve the nonlinear differential equation but assumed that its exact solution is given by a rational equation which includes the cn Jacobian elliptic function and five unknown parameters that need to be determined. Based on his previous results, Elías-Zúñiga obtained the analytical approximate solution of the damped cubic-quintic Duffing oscillator [15] and also developed a "quintication" method [16] to obtain approximate analytical solutions of conservative nonlinear oscillators.

In this paper we obtain the exact expressions for the period and the solution of the undamped, unforced cubic-quintic Duffing oscillator modelled by the second-order nonlinear differential equation $\frac{\mathrm{d}^{2} x}{\mathrm{~d} t^{2}}+a_{1} x+a_{3} x^{3}+a_{5} x^{5}=0$. Unlike the procedure considered by Elías-Zúñiga [14], we do not assume an expression for the solution but solve the nonlinear differential equation exactly. This is done by using elliptic functions so that, after inversion, the solution $x$ is provided as an explicit function of time $t$. The direct symbolic integration performed in this paper can partly be seen as a generalization of the integration for the purely cubic restoring force performed by Starossek [17]. Once the exact expressions for the period and the solution have been obtained, the solutions for the linear, cubic and Duffing oscillators are derived as particular cases of these expressions. Although exact expressions are obtained taking into account that all the coefficients for the first, cubic and fifth terms are positive, under certain conditions these expressions can also apply even if some of these coefficients are negative.

\section{Formulation and solution procedure}

Consider the cubic-quintic nonlinear oscillator, which is modelled by the following secondorder differential equation

$$
\frac{\mathrm{d}^{2} x}{\mathrm{~d} t^{2}}+a_{1} x+a_{3} x^{3}+a_{5} x^{5}=0
$$


with initial conditions

$$
x(0)=A>0 \quad \frac{\mathrm{d} x}{\mathrm{~d} t}(0)=0
$$

In Eq. (1) $x$ and $t$ are generalized dimensionless displacement and time variables, and we assume that the coefficients for the linear and the nonlinear terms satisfy

$$
a_{1} \geq 0 \quad a_{3} \geq 0 \quad a_{5} \geq 0
$$

considering that the three coefficients can not be zero simultaneously. This system corresponds to a nonlinear oscillator for which the nonlinear function $f(x)=a_{1} x+a_{3} x^{3}+a_{5} x^{5}$ is odd, i.e. $f(-x)=-f(x)$ and satisfies $x f(x)>0$ for $x \in[-A, A]$, $x \neq 0$, where $A>0$ is the oscillation amplitude. All the solutions are periodical and the phasespace diagram is made up of an infinite number of closed orbits, each of them for each value of the initial amplitude $A$. This system oscillates around the equilibrium position $x=0$ and the period, $T$, and periodic solution, $x$, are dependent on $A$.

In order to obtain the exact period and periodic solution for Eq. (1), we take into account that this is a conservative system and has the following first integral

$$
\left(\frac{\mathrm{d} x}{\mathrm{~d} t}\right)^{2}+a_{1} x^{2}+\frac{1}{2} a_{3} x^{4}+\frac{1}{3} a_{5} x^{6}=a_{1} A^{2}+\frac{1}{2} a_{3} A^{4}+\frac{1}{3} a_{5} A^{6} \geq 0
$$

which can be written as follows

$$
\left(\frac{\mathrm{d} x}{\mathrm{~d} t}\right)^{2}=a_{1}\left(A^{2}-x^{2}\right)+\frac{1}{2} a_{3}\left(A^{4}-x^{4}\right)+\frac{1}{3} a_{5}\left(A^{6}-x^{6}\right)
$$

From this equation we obtain

$$
\mathrm{d} t= \pm \sqrt{\frac{3}{a_{5}}} \frac{\mathrm{d} x}{\sqrt{\left(A^{2}-x^{2}\right)\left(b_{0}+b_{2} x^{2}+x^{4}\right)}}
$$

where the sign $( \pm)$ is chosen taking into account the sign of $\mathrm{d} x / \mathrm{d} t$ in each quadrant and $b_{0}$ and $b_{2}$ are defined as follows

$$
\begin{gathered}
b_{0}=\frac{3 a_{1}}{a_{5}}+\frac{3 a_{3}}{2 a_{5}} A^{2}+A^{4} \\
b_{2}=\frac{3 a_{3}}{2 a_{5}}+A^{2}
\end{gathered}
$$


Integrating Eq. (6) we obtain

$$
t=\int_{0}^{t} \mathrm{~d} t=-\sqrt{\frac{3}{a_{5}}} \int_{A}^{x} \frac{\mathrm{d} z}{\sqrt{\left(A^{2}-z^{2}\right)\left(b_{0}+b_{2} z^{2}+z^{4}\right)}}=\sqrt{\frac{3}{a_{5}}} \int_{x}^{A} \frac{\mathrm{d} z}{\sqrt{\left(A^{2}-z^{2}\right)\left(b_{0}+b_{2} z^{2}+z^{4}\right)}}
$$

The change of variable $z^{2}=u$ gives

$$
t=\frac{1}{2} \sqrt{\frac{3}{a_{5}}} \int_{x^{2}}^{A^{2}} \frac{\mathrm{d} u}{\sqrt{\left(A^{2}-u\right) u\left(b_{0}+b_{2} u+u^{2}\right)}}
$$

This is an improper integral which contains a square root of a four-degree polynomial in the denominator and so its solution can be expressed as a function of an elliptic integral.

\section{Calculation of the exact period}

The symmetry of the problem indicates that the period of the oscillation $T$ is four times the time taken by the oscillator to go from $u=0$ to $u=A^{2}$. Therefore, from Eq. (10) it follows that

$$
T=2 \sqrt{\frac{3}{a_{5}}} \int_{0}^{A^{2}} \frac{\mathrm{d} u}{\sqrt{\left(A^{2}-u\right) u\left(b_{0}+b_{2} u+u^{2}\right)}}
$$

We consider the definite integral [18, section 3.145, formula 2, pages 270-271]

$$
\int_{\beta}^{y} \frac{\mathrm{d} u}{\sqrt{(\alpha-u)(u-\beta)\left[(u-\sigma)^{2}+\rho^{2}\right]}}=\frac{1}{\sqrt{p q}} F\left(2 \operatorname{arccot} \sqrt{\frac{q(\alpha-y)}{p(y-\beta)}}, \frac{1}{4} \frac{(\alpha-\beta)^{2}-(p-q)^{2}}{p q}\right)
$$

where $\beta<u<\alpha, F(\varphi, m)$ is the incomplete elliptic integral of the first kind defined as follows [19]

$$
F(\phi, m)=\int_{0}^{\phi} \frac{\mathrm{d} \theta}{\sqrt{1-m \sin ^{2} \theta}}
$$

and $p, q$ and $m$ are defined as

$$
\begin{aligned}
& p=\sqrt{(\sigma-\alpha)^{2}+\rho^{2}} \\
& q=\sqrt{(\sigma-\beta)^{2}+\rho^{2}}
\end{aligned}
$$




$$
m=k^{2}=\frac{1}{4} \frac{(\alpha-\beta)^{2}-(p-q)^{2}}{p q}
$$

In Eq. (16), $m$ and $k$ are the parameter and the elliptic modulus, respectively. By comparing the integrals in Eqs. (11) and (12) we obtain $y=A^{2}, \alpha=A^{2}, \beta=0$, as well as the following values for the different parameters which appear in Eq. (12)

$$
\begin{gathered}
\sigma=-\frac{b_{2}}{2}=-\frac{1}{2}\left(\frac{3 a_{3}}{2 a_{5}}+A^{2}\right) \\
\rho=\frac{1}{2} \sqrt{4 b_{0}-b_{2}^{2}}=\frac{1}{2} \sqrt{\frac{12 a_{1}}{a_{5}}-\frac{9 a_{3}^{2}}{4 a_{5}^{2}}+\frac{3 a_{3}}{a_{5}} A^{2}+3 A^{4}} \\
p=\sqrt{b_{0}+b_{2} A^{2}+A^{4}}=\sqrt{\frac{3}{a_{5}}} \sqrt{a_{1}+a_{3} A^{2}+a_{5} A^{4}} \\
q=\sqrt{b_{0}}=\sqrt{\frac{3 a_{1}}{a_{5}}+\frac{3 a_{3}}{2 a_{5}} A^{2}+A^{4}} \\
m=\frac{A^{4}-\sqrt{b_{0}+b_{2} A^{2}+A^{4}}+\sqrt{b_{0}}}{4 \sqrt{b_{0}} \sqrt{b_{0}+b_{2} A^{2}+A^{4}}}=\frac{1}{4}\left(2-\sqrt{\frac{3}{2}} \frac{4 a_{1}+3 a_{3} A^{2}+2 a_{5} A^{4}}{\sqrt{\left(a_{1}+a_{3} A^{2}+a_{5} A^{4}\right)\left(6 a_{1}+3 a_{3} A^{2}+2 a_{5} A^{4}\right)}}\right)
\end{gathered}
$$

As $y=\alpha=A^{2}$, then $F(2 \operatorname{arccot} 0, m)=F(\pi, m)=2 K(m)$, where $K(m)$ is the complete elliptic integral of the first kind defined as [19]

$$
K(m)=\int_{0}^{\pi / 2} \frac{\mathrm{d} \theta}{\sqrt{1-m \sin ^{2} \theta}}
$$

From Eqs. (11)-(22) we conclude that the exact period of the undamped, unforced cubicquintic nonlinear oscillator can be written in the compact form

$$
T=4\left(\frac{6}{q_{1} q_{2}}\right)^{1 / 4} K(m)
$$

where $m$ is given as

$$
m=\frac{1}{2}-\frac{q_{3}}{4} \sqrt{\frac{3}{2 q_{1} q_{2}}}
$$

and $q_{1}, q_{2}$ and $q_{3}$-which depend on $a_{1}, a_{3}, a_{5}$ and $A$ - are defined as follows 


$$
\begin{gathered}
q_{1}=a_{1}+a_{3} A^{2}+a_{5} A^{4} \\
q_{2}=6 a_{1}+3 a_{3} A^{2}+2 a_{5} A^{4} \\
q_{3}=4 a_{1}+3 a_{3} A^{2}+2 a_{5} A^{4}
\end{gathered}
$$

As mentioned above we consider the signs shown in Eq. (2) for $a_{1}, a_{3}$ and $a_{5}$. However, other combinations of signs are possible for these coefficients provided they lead to positive real values for the period $T$. In particular, from Eqs. (23) and (24) it is required that $q_{1} q_{2}>0$.

\section{Calculation of the exact periodic solution}

From Eq. (6) we can obtain $t$ as a function of $x$ for the following cases:

(a) Trajectory $1 \rightarrow 2(0 \leq t \leq T / 4$ and $A \geq x \geq 0), x>0$ and $\mathrm{d} x / \mathrm{d} t<0$.

(b) Trajectory $2 \rightarrow 3(T / 4 \leq t \leq T / 2$ and $0 \geq x \geq-A), x<0$ and $\mathrm{d} x / \mathrm{d} t<0$.

(c) Trajectory $3 \rightarrow 4(T / 2 \leq t \leq 3 T / 4$ and $-A \leq x \leq 0), x<0$ and $\mathrm{d} x / \mathrm{d} t>0$.

(d) Trajectory $4 \rightarrow 1(3 T / 4 \leq t \leq T$ and $0 \leq x \leq A), x>0$ and $\mathrm{d} x / \mathrm{d} t>0$.

From Eq. (6), and taking into account the change of variable $z^{2}=u$, it follows that for trajectory $1 \rightarrow 2$ we have

$$
2 \sqrt{\frac{a_{5}}{3}} \int_{0}^{t} \mathrm{~d} t=2 \sqrt{\frac{a_{5}}{3}} t=\int_{x^{2}}^{A^{2}} \frac{\mathrm{d} u}{\sqrt{\left(A^{2}-u\right) u\left(b_{0}+b_{2} u+u^{2}\right)}}
$$

The definite integral in Eq. (28) can be split as follows

$$
2 \sqrt{\frac{a_{5}}{3}} t=\int_{0}^{A^{2}} \frac{\mathrm{d} u}{\sqrt{\left(A^{2}-u\right) u\left(b_{0}+b_{2} u+u^{2}\right)}}-\int_{0}^{x^{2}} \frac{\mathrm{d} u}{\sqrt{\left(A^{2}-u\right) u\left(b_{0}+b_{2} u+u^{2}\right)}}
$$

The values of the two integrals on the right-hand side of Eq. (29) can be calculated taking into account Eqs. (11) and (12) and their values are

$$
\begin{gathered}
\int_{0}^{A^{2}} \frac{\mathrm{d} u}{\sqrt{\left(A^{2}-u\right) u\left(b_{0}+b_{2} u+u^{2}\right)}}=2 \sqrt{\frac{a_{5}}{3}} \frac{T}{4} \\
\int_{0}^{x^{2}} \frac{\mathrm{d} u}{\sqrt{\left(A^{2}-u\right) u\left(b_{0}+b_{2} u+u^{2}\right)}}=\frac{1}{\sqrt{p q}} F\left(2 \operatorname{arccot} \sqrt{\frac{q\left(A^{2}-x^{2}\right)}{p x^{2}}}, m\right)
\end{gathered}
$$


Substituting Eqs. (35) and (36) into Eq. (29) gives

$$
2 \sqrt{p q} \sqrt{\frac{a_{5}}{3}}\left(\frac{T}{4}-t\right)=F\left(2 \operatorname{arccot} \sqrt{\frac{q\left(A^{2}-x^{2}\right)}{p x^{2}}}, m\right)
$$

and using Eqs. (19), (20), (23), (25), (26) and (27), Eq. (32) can be written as

$$
2 K(m)-\left(\frac{8 q_{1} q_{2}}{3}\right)^{1 / 4} t=F\left(2 \operatorname{arccot}\left[\left(\frac{q_{2}}{6 q_{1}}\right)^{1 / 4}\left(\frac{A^{2}-x^{2}}{x^{2}}\right)^{1 / 2}\right], m\right)
$$

The inverse function of $F(\varphi, m)$ is the Jacobi amplitude $\varphi[19,20]$

$$
F^{-1}(u, m)=\phi=\operatorname{am}(u, m)
$$

whose cosine is the Jacobi cosine function, cn [20]

$$
\cos \varphi=\cos (\operatorname{am}(u, m))=\operatorname{cn}(u, m)
$$

In order to introduce an "arccos" function in Eq. (14) we take into account that

$$
2 \operatorname{arccot} z=\phi
$$

where

$$
z=\left(\frac{q_{2}}{6 q_{1}}\right)^{1 / 4}\left(\frac{A^{2}-x^{2}}{x^{2}}\right)^{1 / 2}
$$

From Eq. (35) we obtain

$$
\tan ^{2} \frac{\phi}{2}=\frac{1}{z^{2}}
$$

Taking into account the relation

$$
\tan ^{2} \frac{\phi}{2}=\frac{1-\cos \phi}{1+\cos \phi}
$$

we can finally write

$$
\cos \phi=\frac{z^{2}-1}{z^{2}+1}=\frac{\sqrt{q_{2}}\left(A^{2}-x^{2}\right)-\sqrt{6 q_{1}} x^{2}}{\sqrt{q_{2}}\left(A^{2}-x^{2}\right)+\sqrt{6 q_{1}} x^{2}}
$$


which allows us to write Eq. (14) as follows

$$
2 K(m)-\left(\frac{8 q_{1} q_{2}}{3}\right)^{1 / 4} t=F\left(\arccos \left(\frac{\sqrt{q_{2}}\left(A^{2}-x^{2}\right)-\sqrt{6 q_{1}} x^{2}}{\sqrt{q_{2}}\left(A^{2}-x^{2}\right)+\sqrt{6 q_{1}} x^{2}}\right), m\right)
$$

and from Eqs. (34), (35) and (41) we can write, after some simplifications

$$
\frac{\sqrt{q_{2}}\left(A^{2}-x^{2}\right)-\sqrt{6 q_{1}} x^{2}}{\sqrt{q_{2}}\left(A^{2}-x^{2}\right)+\sqrt{6 q_{1}} x^{2}}=\operatorname{cn}\left(2 K(m)-\left(\frac{8}{3} q_{1} q_{2}\right)^{1 / 4} t, m\right)=-\operatorname{cn}\left(\left(\frac{8}{3} q_{1} q_{2}\right)^{1 / 4} t, m\right)
$$

where the relation $\operatorname{cn}(2 K(m)-u, m)=-\operatorname{cn}(u, m)$ [20] has been taken into account.

Finally we can write

$$
x_{1}(t)=A\left[1+\sqrt{\frac{6 q_{1}}{q_{2}}}\left(\frac{1-\operatorname{cn}\left(\left(\frac{8}{3} q_{1} q_{2}\right)^{1 / 4} t, m\right)}{1+\operatorname{cn}\left(\left(\frac{8}{3} q_{1} q_{2}\right)^{1 / 4} t, m\right)}\right)\right]^{-1 / 2}
$$

which is valid for $0 \leq t \leq T / 4$.

From Eq. (6), it follows for trajectory $2 \rightarrow 3$

$$
2 \sqrt{\frac{a_{5}}{3}} \int_{T / 4}^{t} \mathrm{~d} t=2 \sqrt{\frac{a_{5}}{3}}\left(t-\frac{T}{4}\right)=-\int_{0}^{x^{2}} \frac{\mathrm{d} u}{\sqrt{\left(A^{2}-u\right) u\left(b_{0}+b_{2} u+u^{2}\right)}}
$$

and for trajectory $3 \rightarrow 4(T / 4 \leq t \leq T / 2)$

$$
2 \sqrt{\frac{a_{5}}{3}} \int_{T / 2}^{t} \mathrm{~d} t=2 \sqrt{\frac{a_{5}}{3}}\left(t-\frac{T}{2}\right)=\int_{A^{2}}^{x^{2}} \frac{\mathrm{d} u}{\sqrt{\left(A^{2}-u\right) u\left(b_{0}+b_{2} u+u^{2}\right)}}
$$

Proceeding in the same manner as for trajectory $1 \rightarrow 2$, it is follows that $x_{2}(t)=-x_{1}(t)$ which is valid for $T / 4 \leq t \leq 3 T / 4$, because $x<0$ for these values of time.

Finally, for trajectory $4 \rightarrow 1$ we have

$$
2 \sqrt{\frac{a_{5}}{3}} \int_{3 T / 4}^{t} \mathrm{~d} t=2 \sqrt{\frac{a_{5}}{3}}\left(t-\frac{3 T}{4}\right)=\int_{0}^{x^{2}} \frac{\mathrm{d} u}{\sqrt{\left(A^{2}-u\right) u\left(b_{0}+b_{2} u+u^{2}\right)}}
$$

and we obtain the same value for the solution as that given in Eq. (43).

The exact solution of the undamped, unforced cubic-quintic oscillator can be written as follows 


$$
x(t)= \begin{cases}x_{1}(t) & 0 \leq t \leq T / 4 \\ -x_{1}(t) & T / 4 \leq t \leq 3 T / 4 \\ x_{1}(t) & 3 T / 4 \leq t \leq T\end{cases}
$$

Taking into account the relation [20, formula 16.18.4, page 574]

$$
\frac{1-\operatorname{cn} 2 u}{1+\operatorname{cn} 2 u}=\frac{\operatorname{sn}^{2} u \operatorname{dn}^{2} u}{\operatorname{cn}^{2} u}
$$

and Eqs. (43) and (47), the exact periodic solution of undamped, unforced the cubic-quintic oscillator can be can be written in compact form as follows

$$
x(t)=\frac{A \operatorname{cn}\left(\left(\frac{1}{6} q_{1} q_{2}\right)^{1 / 4} t, m\right)}{\sqrt{\operatorname{cn}^{2}\left(\left(\frac{1}{6} q_{1} q_{2}\right)^{1 / 4} t, m\right)+\left(\frac{6 q_{1}}{q_{2}}\right)^{1 / 2} \operatorname{sn}^{2}\left(\left(\frac{1}{6} q_{1} q_{2}\right)^{1 / 4} t, m\right) \operatorname{dn}^{2}\left(\left(\frac{1}{6} q_{1} q_{2}\right)^{1 / 4} t, m\right)}}
$$

which is valid for all values of $t$ and where cn, sn and $\mathrm{dn}$ are the basic Jacobi elliptic functions [20].

\section{Particular cases}

In Eq. (3) we assumed the condition that coefficients $a_{1}, a_{3}$ and $a_{5}$ must satisfy. However, Eqs. (23) and (43) can be applied for other values of these coefficients as we shall now show.

Firstly we consider the linear oscillator which satisfies

$$
a_{1}>0 \quad a_{3}=0 \quad a_{5}=0
$$

From Eqs. (25)-(27) we obtain $q_{1}=a_{1}, q_{2}=6 a_{1}$ and $q_{3}=4 a_{1}$, and substituting these values in Eq. (24) we obtain $m=0$. From Eq. (23), and taking into account that $K(0)=\pi / 2$, we obtain

$$
T=\frac{4}{\sqrt{a_{1}}} K(0)=\frac{2 \pi}{\sqrt{a_{1}}}
$$

Substituting Eq. (50) into Eqs. (43) and (46) and taking into account that $\operatorname{cn}(u, 0)=\cos u[20]$, we can write

$$
x(t)= \pm A\left(1+\frac{1-\operatorname{cn}\left(2 \sqrt{a_{1}} t, 0\right)}{1+\operatorname{cn}\left(2 \sqrt{a_{1}} t, 0\right)}\right)^{-1 / 2}= \pm A\left(1+\frac{1-\cos \left(2 \sqrt{a_{1}} t, 0\right)}{1+\cos \left(2 \sqrt{a_{1}} t, 0\right)}\right)^{-1 / 2}
$$

and considering the relation 


$$
\tan ^{2} \alpha=\frac{1-\cos 2 \alpha}{1+\cos 2 \alpha}
$$

we finally obtain

$$
x(t)= \pm \frac{A}{\sqrt{1+\tan ^{2}\left(\sqrt{a_{1}} t\right)}}=A \cos \left(\sqrt{a_{1}} t\right)
$$

As can be seen, Eqs. (51) and (54) are the period and the solution of the linear oscillator

$$
\frac{\mathrm{d}^{2} x}{\mathrm{~d} t^{2}}+a_{1} x=0
$$

with the initial conditions given in Eq. (2).

Now we consider the following particular case

$$
a_{1} \geq 0 \quad a_{3}>0 \quad a_{5}=0
$$

which corresponds to the Duffing oscillator with cubic nonlinearity. Substituting Eq. (56) into Eq. (23) we obtain

$$
T=4\left(\frac{6}{\left(a_{1}+a_{3} A^{2}\right)\left(6 a_{1}+3 a_{3} A^{2}\right)}\right)^{1 / 4} K\left(-\frac{1}{4}\left(\frac{\sqrt{3}\left(4 a_{1}+3 a_{3} A^{2}\right)}{\sqrt{2\left(a_{1}+a_{3} A^{2}\right)\left(6 a_{1}+3 a_{3} A^{2}\right)}}-2\right)\right)
$$

It is well known that the exact period for the Duffing oscillator with cubic nonlinearity is given by the following equation [2]

$$
T=\frac{4}{\sqrt{a_{1}+a_{3} A^{2}}} K\left(\frac{a_{3} A^{2}}{2\left(a_{1}+a_{3} A^{2}\right)}\right)
$$

which is formally different from Eq. (57). However, Eqs. (57) and (58) are equivalent as we shall demonstrate. From Abramowitz and Stegun [19], formula 17.3.29, page 591, the following relation can be written

$$
K\left(m_{1}\right)=\frac{2}{1+\sqrt{1-m_{1}}} K\left(\left(\frac{1-\sqrt{1-m_{1}}}{1+\sqrt{1-m_{1}}}\right)^{2}\right)
$$

where $K(m)$ is defined in Eq. (22) and $m_{1}$ is the parameter of the complete elliptic function of the first kind which appears in Eq. (58), i.e. 


$$
m_{1}=\frac{a_{3} A^{2}}{2\left(a_{1}+a_{3} A^{2}\right)}
$$

Taking into account Eqs. (59) and (60), and after some mathematical operations, Eq. (58) can be written as follows

$$
T=\frac{16}{2 \sqrt{a_{1}+a_{3} A^{2}}+\sqrt{4 a_{1}+2 a_{3} A^{2}}} K\left(\frac{4 a_{1}+3 a_{3} A^{2}-2 \sqrt{4 a_{1}^{2}+6 a_{1} a_{3} A^{2}+2 a_{3}^{2} A^{4}}}{4 a_{1}+3 a_{3} A^{2}+2 \sqrt{4 a_{1}^{2}+6 a_{1} a_{3} A^{2}+2 a_{3}^{2} A^{4}}}\right)
$$

Once again, from Abramowitz and Stegun [19], formula 17.4.17 (negative parameter), page 593, we can write

$$
K\left(-m_{2}\right)=\frac{2}{\sqrt{1+m_{2}}} K\left(\frac{m_{2}}{1+m_{2}}\right)
$$

where $m_{2}>0$. From this equation it is possible to obtain

$$
K\left(\frac{m_{2}}{1+m_{2}}\right)=\frac{1}{2} \sqrt{1+m_{2}} K\left(-m_{2}\right)
$$

From Eq. (61) we define

$$
\frac{m_{2}}{1+m_{2}}=\frac{4 a_{1}+3 a_{3} A^{2}-2 \sqrt{4 a_{1}^{2}+6 a_{1} a_{3} A^{2}+2 a_{3}^{2} A^{4}}}{4 a_{1}+3 a_{3} A^{2}+2 \sqrt{4 a_{1}^{2}+6 a_{1} a_{3} A^{2}+2 a_{3}^{2} A^{4}}}
$$

which allows $m_{2}$ to be obtained as follows

$$
m_{2}=\frac{1}{4}\left(\frac{\sqrt{3}\left(4 a_{1}+3 a_{3} A^{2}\right)}{\sqrt{2\left(a_{1}+a_{3} A^{2}\right)\left(6 a_{1}+3 a_{3} A^{2}\right)}}-2\right)
$$

From Eqs. (61), (63) and (65) we finally obtain

$$
\begin{aligned}
& T=\frac{16}{2 \sqrt{a_{1}+a_{3} A^{2}}+\sqrt{4 a_{1}+2 a_{3} A^{2}}} \frac{1}{2} \sqrt{1+m_{2}} K\left(-m_{2}\right)= \\
& =4\left(\frac{6}{\left(a_{1}+a_{3} A^{2}\right)\left(6 a_{1}+3 a_{3} A^{2}\right)}\right)^{1 / 4} K\left(-\frac{1}{4}\left(\frac{\sqrt{3}\left(4 a_{1}+3 a_{3} A^{2}\right)}{\sqrt{2\left(a_{1}+a_{3} A^{2}\right)\left(6 a_{1}+3 a_{3} A^{2}\right)}}-2\right)\right)
\end{aligned}
$$

which is the period given in Eq. (57). This means that Eq. (57) provides the exact period of the cubic Duffing oscillator. To obtain the solution for the undamped, unforced Duffing oscillator with cubic nonlinearity, we substitute Eq. (56) into Eqs. (43) and (48) and obtain 


$$
\left.x(t)= \pm A\left[1+\sqrt{\frac{2\left(a_{1}+a_{3} A^{2}\right)}{2 a_{1}+a_{3} A^{2}}}\left(\frac{1-\operatorname{cn}\left(2^{3 / 4} 3^{-1 / 4}\left[\left(a_{1}+a_{3} A^{2}\right)\left(6 a_{1}+3 a_{3} A^{2}\right)\right]^{/ 4} t,-m_{2}\right.}{1+\operatorname{cn}\left(2^{3 / 4} 3^{-1 / 4}\left[\left(a_{1}+a_{3} A^{2}\right)\left(6 a_{1}+3 a_{3} A^{2}\right)\right]^{1 / 4} t,-m_{2}\right.}\right)\right)\right]^{-1 / 2}
$$

where $m_{2}$ is given in Eq. (65). The exact solution for the Duffing oscillator is [2]

$$
x(t)=A \operatorname{cn}\left(\sqrt{a_{1}+a_{3} A^{2}} t, \frac{a_{3} A^{2}}{2\left(a_{1}+a_{3} A^{2}\right)}\right)
$$

Eqs. (67) and (68) give the same result and the two equations are equivalent; however, it is much more complex to demonstrate this equivalence than it is to demonstrate the equivalence between the periods given in Eqs. (57) and (58) and we have to admit that we were not able to do so. Nevertheless, we believe it is possible to demonstrate this equivalence using Eq. (68) and taking into account the descending Landen transformation (Gauss' transformation) [19, formula 16.12, page 573], the change of parameter (negative parameter) [19, formula 16.10 , page 573$]$ as well as the different relations between the Jacobi elliptic functions.

Finally we consider the following set of parameters

$$
a_{1} \geq 0 \quad a_{3}=0 \quad a_{5}>0
$$

which corresponds to the quintic Duffing oscillator. This oscillator was approximately solved using a cubication method [10] and exactly solved for $a_{1}=a_{5}=1$ [12]. From Eqs. (23)-(27) and taking into account the parameters in Eq. (69), we can obtain the exact period for the undamped, unforced quintic Duffing oscillator

$$
T=4\left(\frac{3}{\left(a_{1}+a_{5} A^{4}\right)\left(3 a_{1}+a_{5} A^{4}\right)}\right)^{1 / 4} K\left(\frac{1}{4}\left(2-\frac{\sqrt{3}\left(2 a_{1}+a_{5} A^{4}\right)}{\sqrt{\left(a_{1}+a_{5} A^{4}\right)\left(2 a_{1}+a_{5} A^{4}\right)}}\right)\right)
$$

\section{Some simulations}

Knowing the exact expressions for the period and the solution of the undamped, unforced cubic-quintic oscillator allows us to determine in the detail the behaviour of this nonlinear oscillator as a function of $a_{1}, a_{3}, a_{5}$ and $A$. In this section we present some numerical results corresponding to the period and the solution for different values of parameters $a_{1}, a_{3}$ and $a_{5}$, as well as the oscillation amplitude $A$. 
As we pointed out in section 5, the exact period and solution given in Eqs. (23), (43) and (49) are valid for all values of parameters $a_{1}, a_{3}$ and $a_{5}$ satisfying $a_{1} \geq 0, a_{3} \geq 0$ and $a_{5}>0$. Figure 1 shows the variation of the period as a function of $A$ for $a_{1}=a_{3}=1$ and three values of $a_{5}$ (5, 10 and 30). As can be seen, the period decreases when $A$ increases and the maximum value for the period is $2 \pi$ which is obtained when $A \rightarrow 0$ (linear oscillator). On the other hand, for oscillators with strong nonlinearity (large values of $a_{5}$ ), the period decreases more quickly than for oscillators with less nonlinearity. Figure 2 shows the values for parameter $m$ (Eq. (24)) as a function of $A$ for $a_{1}=a_{3}=1$ and three values of $a_{5}(5,10$ and 30). For large values of $a_{5}$ and $A$, all curves for parameter $m$ tend to the same value. From Eq. (24) we obtain

$$
\lim _{A \rightarrow \infty} m=\frac{2-\sqrt{3}}{4} \approx 0.0669873
$$

and from Eq. (23), we can do the following series expansion for large values of $A$

$$
T \approx \frac{3^{1 / 4} 4}{\sqrt{a_{5}} A^{2}} K\left(\frac{2-\sqrt{3}}{4}\right) \approx \frac{8.41309}{\sqrt{a_{5}} A^{2}}
$$

which is the period for the truly nonlinear "quintic" oscillator [21]

$$
\frac{\mathrm{d}^{2} x}{\mathrm{~d} t^{2}}+a_{5} x^{5}=0
$$

and satisfies that

$$
\lim _{\sqrt{a_{5}} A^{2} \rightarrow \infty} \sqrt{a_{5}} A^{2} T \approx 8.41309
$$

The results obtained in this paper are always valid for $a_{1} \geq 0, a_{3} \geq 0$ and $a_{5} \geq 0$. However, the exact expressions for the period and solution can also be used when one or two of these coefficients are negative, depending on the values of these coefficients and the oscillation amplitude.

We shall now analyse the set of parameters $a_{1}=1, a_{3}=-\sqrt{40} \approx-6.324555$ and $a_{5}=10$ which gives us the following value for parameter $q_{1}$ (Eq. (25)) or the nonlinear function $f(x)=x-\sqrt{40} x^{3}+10 x^{5}$

$$
f(A)=q_{1}(A)=A-\sqrt{40} A^{3}+10 A^{5}
$$


From this equation it is easy to verify that $f(A)=q_{1}(A)=0$ for $A=0$ and $A=10^{-1 / 4} \approx 0.562$. Figure 3 shows the variation of the nonlinear function $f$ (or parameter $q_{1}$ ) as a function of the initial amplitude $A$ for (a) $a_{1}=1, a_{3}=-\sqrt{40}$ and $a_{5}=10$, and for (b) the quintic oscillator $\left(a_{1}=1, a_{3}=0\right.$ and $\left.a_{5}=10\right)$. As can be seen, in the case of the quintic oscillator, the function $f$ increases as $A$ increases. However, if $a_{3}=-\sqrt{40}$ the effect of the cubic term is to make $f$ increase initially since the linear term prevails over the cubic term, then as $A$ continues to increase, the linear function $f$ (and therefore $q_{1}$ ) begins to decrease until it is cancelled out when $A=10^{-1 / 4}$. In this case the movement is no longer oscillatory and it is easy to verify that the period $T$ in Eq. (23) is not defined for $A=10^{-1 / 4}$. To demonstrate this, in Figure 4 we plotted the variation of the period as a function of $A$ for $a_{1}=1, a_{5}=10$ and five values of $a_{3}=0,-3,-5$ and $-\sqrt{40}$. As can be seen, the behaviour of the period is different to that shown in Figure 1. In fact, for $a_{3}<0$, when $A$ increases the period also increases, reaches a maximum value, and then decreases. For $a_{1}=1$ and $a_{5}=10$, the exact equations for the period and the solution are applicable for all values of $A$ only if $a_{3}>-\sqrt{40}$, as we can see in Figure 5 in which the white zone is a "forbidden" area because for these values of $a_{3}$ and $A$ the period does not take real values but a complex or infinite value. In Figures 6 and 7 we plot the period $T$ and the parameter $m$, respectively, as a function of $A$ for parameters $a_{1}=1$, $a_{3}=-\sqrt{40}$ and $a_{5}=10$. In Figure 7 we can see that $m$ is discontinuous when $A=10^{-1 / 4}$ and it is satisfied that

$$
\begin{aligned}
& \lim _{\substack{A \rightarrow 10^{-1 / 4} \\
A<10^{-1 / 4}}} m(A)=\frac{1}{4}(2-\sqrt{3}) \approx 0.0669873 \\
& \lim _{\substack{A \rightarrow 10^{-1 / 4} \\
A>10^{-1 / 4}}} m(A)=\frac{1}{4}(2+\sqrt{3}) \approx 0.933013
\end{aligned}
$$

Now we shall analyse the behaviour of the cubic-quintic oscillator for $a_{1}=1$ and $a_{5}=10$ and a value of $a_{3}$ close to but slightly lower than $-\sqrt{40} \approx-6.324555$. In particular, we consider $a_{3}=-6.324$. Figures 8 and 9 show the period and parameter $\mathrm{m}$ as a function of the initial amplitude. Now the period reaches a maximum value of 88.73 for $A=0.564$ and the parameter $m$, whose behaviour is very different to that shown in Figure 2, shows an abrupt variation around $A \approx 0.55$ but, as compared with Figure 7 , it is no longer a discontinuous function. In Figure 10 we plot the period as a function of $a_{3}$ and $A$ for $a_{1}=1, a_{3} \geq-6.324$ and 
$a_{5}=10$. Figures 11 and 12 show the exact solution and the exact velocity for $a_{1}=1, a_{3}=$ -6.324 and $a_{5}=10$, and for values of $A, 0.1,0.5,0.564$ (at which the period is maximum), $0.65,0.725$ and 1 , respectively. The effect of parameter $a_{3}$ when it is $<0$ is clearly seen in these figures. Comparing Figure 11 (a) in which the linear term prevails with Figure 11 (f) in which the quintic term prevails, if $a_{3}$ were also positive, as $A$ increased the sinusoidal function in (a) would contract until it reached the curve shown in (f). However, the effect of parameter $a_{3}=-6.324$ means that for an interval of values of $A$ the sinusoidal function in (a) begins to widen until it approximates a crenel function and then decreases as the quintic term begins to prevail over the other two. As can be seen in Figure 13, the effect is even more interesting in the case of velocity. The phase plot for $a_{1}=1, a_{3}=-6.324$ and $a_{5}=10$ and for $A=0.1,0.5,0.75$ and 1 is shown in Figure 13.

Finally, since the nonlinear function in Eq. (1) is an odd function of $x$, the periodic solution $x(t)$ in Eq. (49) can be represented by a Fourier series containing only odd multiples of $2 \pi t / T$, i.e.

$$
x(t)=A \sum_{n=0}^{\infty} c_{2 n+1} \cos \left[(2 n+1) \frac{2 \pi t}{T}\right]
$$

where

$$
c_{2 n+1}=\frac{2}{T} \int_{0}^{T} \frac{x(t)}{A} \cos \left[(2 n+1) \frac{2 \pi t}{T}\right] \mathrm{d} t=\frac{2}{T} \int_{0}^{T / 4} \frac{x_{1}(t)}{A} \cos \left[(2 n+1) \frac{2 \pi t}{T}\right] \mathrm{d} t
$$

This equation allows us to obtain the values of the coefficients $c_{2 n+1}$ of the Fourier series expansion of the exact periodic solution $x(t)$. The integral in Eq. (79) must be computed numerically and this was done with the help of symbolic computation software such as MATHEMATICA. Figure 14 shows the first four Fourier coefficients $c_{1}, c_{3}, c_{5}$, and $c_{7}$ as a function of $A$ for $a_{1}=1, a_{3}=-6.324$ and $a_{5}=10$. In this figure horizontal dashed lines correspond to $c_{1}=1, c_{3}=0$ and $c_{7}=0$. Once again, it can be seen that the oscillator has a strange behaviour close to $A \approx 0.55$. On the other hand, when $A$ tends to infinity these Fourier coefficients (which correspond to the truly nonlinear "quintic" oscillator, Eq. (73)) are positive and their values are

$$
\begin{aligned}
& \lim _{A \rightarrow \infty} c_{1} \approx 0.926430 \\
& \lim _{A \rightarrow \infty} c_{3} \approx 0.0633949
\end{aligned}
$$




$$
\begin{aligned}
& \lim _{A \rightarrow \infty} c_{5} \approx 0.00877599 \\
& \lim _{A \rightarrow \infty} c_{7} \approx 0.000172526
\end{aligned}
$$

which coincide with the values that can be numerically calculated [21]. However, for other values of $A$ some of these Fourier coefficients take negative values and this fact explains the different behaviour of the exact solution which can be seen in Figure 11. In fact, from Figure 14 and Eqs. (80)-(83) we conclude that $c_{1}>0$ and $c_{5}>0$ for all values of $A$, whereas $c_{3}<0$ for $A<0.7257$ and $c_{7}<0$ for $A<0.7123$. On the other hand, $c_{1}>1$ for $A<0.6872$. Finally, Table 1 shows the values of the four Fourier coefficients of the series expansion of the exact solution corresponding to Figure 11.

\section{Conclusions}

The period and exact solution of the undamped, unforced nonlinear cubic-quintic Duffing oscillator have been obtained. Unlike Elías-Zúñiga's procedure [14], we do not assume any expression for the solution but exactly solve the nonlinear differential equation, which allows us to obtain the period and, after inversion, the solution for this conservative nonlinear oscillator. The exact period is given in terms of a complete elliptic integral of the first kind and the solution is a piecewise function involving cn Jacobi elliptic functions which is valid for all periods of the motion. Some particular cases are analysed and discussed and the Fourier series expansion of the exact solution has been calculated for some of them. Knowing the Fourier coefficients is useful for examining resonance phenomena under periodic or quasiperiodic forcing [4]. Our future work will focus on investigating cubicquintic Duffing oscillators which have more than one equilibrium point, in particular, to obtain the period and the exact solution for periodic motions around an equilibrium point other than $(0,0)$. In this case, the maximum displacements to the right and to the left of the equilibrium point are not of the same magnitude. Finally, the exact expressions obtained is this paper may be used in a "quintication" method based on the Chebyshev expansion of the restoring force $[16,22]$ to obtain highly accurate analytical approximate solutions for conservative nonlinear oscillators. 


\section{Acknowledgements}

This work was supported by the "Generalitat Valenciana" of Spain, under project PROMETEOII/2015/015, and by the "Vicerrectorado de Tecnologías de la Información" of the University of Alicante, Spain, under project GITE-09006-UA. 


\section{References}

[1] A. H. Nayfeh, Problems in Perturbation, John Wiley \& Sons, New York, 1985.

[2] R. E. Mickens, Oscillations in Planar Dynamics Systems, World Scientific, Singapore, 1996.

[3] A. H. Nayfeh and D. T. Mook, Nonlinear Oscillations, Wiley-Interscience, New York, 1979.

[4] S. K. Lai, C. W. Lim, B. S. Wu, C. Wang, Q. C. Zeng, and X. F. He, "Newtonharmonic balancing approach for accurate solutions to nonlinear cubic-quintic Duffing oscillators," Applied Mathematical Modelling, vol. 33, pp. 852-866, 2009. http://dx.doi.org/10.1016/j.apm.2007.12.012

[5] C. W. Lim, R. Xu, S. K. Lai, Y. M. Yu, and Q. Yang, "Nonlinear free vibration of an elastically-restrained beam with a point mass via the Newton-harmonic balancing approach,” International Journal of Nonlinear Sciences and Numerical Simulation, vol. 10, pp. 661-674, 2009. http://dx.doi.org/10.1515/IJNSNS.2009.10.5.661

[6] J. I. Ramos, "On Linstedt-Poincaré technique for the quintic Duffing equation," Applied Mathematics and Computation, vol. 193, pp. 303-310, 2007. http://dx.doi.org/10.1016/j.amc.2007.03.050

[7] J. Lin, "A new approach to Duffing equation with strong and high order nonlinearity," Communications in Nonlinear Science and Numerical Simulation, vol. 4, pp. 132-135, 2009. http://dx.doi.org/10.1016/S1007-5704(99)90026-6

[8] T. Pirbodaghi, S. H. Hoseini, M. T. Ahmadian, and G. H. Farrahi, "Duffing equations with cubic and quintic nonlinearities," Computers and Mathematics with Applications, vol. 57, pp. 500-506, 2009. http://dx.doi.org/10.1016/j.camwa.2008.10.082

[9] B. S. Wu, W. P. Sun, and C. W. Lim, "An analytical approximate technique for a class of strongly non-linear oscillators," International Journal of Non-Linear Mechanics, vol. 41, pp. 766-774, 2009. http://dx.doi.org/10.1016/j.ijnonlinmec.2006.01.006

[10] A. Beléndez, G. Bernabeu, J. Francés, D. I. Méndez, and S. Marini, “An accurate closed-form approximate solution for the quintic Duffing oscillator equation," 
Mathematical and Computer Modelling, vol. 52, pp. 637-641, 2010. http://dx.doi.org/10.1016/j.mcm.2010.04.010

[11] A. Beléndez, M. L. Alvarez, J. Francés, S. Bleda, T. Beléndez, A. Nájera and E. Arribas, "Analytical approximate solutions for the cubic-quintic Duffing oscillator in terms of elementary functions," Journal of Applied Mathematics, vol. 2012, ID 286290, 16 pages, 2012. http://dx.doi.org/10.1155/2012/286290

[12] G. M. Scarpello, "Exact solution to a first-fifth power nonlinear unforced oscillator," Applied Mathematical Sciences, vol. 4, 3589-3594, 2010.

[13] V. Chua, "Cubic-quintic Duffing oscillators," December 2003. http://citeseerx.ist.psu.edu/viewdoc/summary?doi=10.1.1.585.9915

[14] A. Elías-Zúñiga, "Exact solution of the cubic-quintic Duffing oscillator," Applied Mathematical Modelling, vol. 37, 2574-2579, 2013. http://dx.doi.org/10.1016/j.apm.2012.04.005

[15] A. Elías-Zúñiga, "Solution of the damped cubic-quintic Duffing oscillator by using Jacobi elliptic functions," Applied Mathematics and Computation, vol. 246, pp. 474 481, 2014. http://dx.doi.org/10.1016/j.amc.2014.07.110

[16] A. Elías-Zúñiga, “"Quintication” method to obtain approximate analytical solutions of non-linear oscillators," Applied Mathematics and Computation, vol. 243, pp. 849-855, 2014. http://dx.doi.org/10.1016/j.amc.2014.05.085

[17] U. Starossek, "A low-frequency pendulum mechanism," Mechanism and Machine Theory, vol. 83, pp. 81-90 (2015).

http://dx.doi.org/10.1016/j.mechmachtheory.2014.08.010

[18] I. S. Gradshteyn and I. M. Ryzhik, Table of Integrals, Series and Products. Sixth Edition. San Diego, Academic Press, 2000.

[19] L. M. Milne-Thomson, "Elliptic integrals", Ch. 17 in Handbook of Mathematical functions with Formulas, Graphics and Mathematical Tables. M. Abramowitz and I. A. Stegun (Eds.). New York, Dover, pp. 587-607, 1972. 
[20] L. M. Milne-Thomson, "Jacobian elliptic functions and theta functions", Ch. 16 in Handbook of Mathematical functions with Formulas, Graphics and Mathematical Tables. M. Abramowitz and I. A. Stegun (Eds.). New York, Dover, pp. 567-581, 1972.

[21] A. Beléndez, J. Francés, T. Beléndez, S. Bleda, C. Pascual, and E. Arribas, "Nonlinear oscillator with power-form elastic-term: Fourier series expansion of the exact solution," Communications in Nonlinear Science and Numerical Simulation, vol. 22, pp. 134-148, 2015. http://dx.doi.org/10.1016/j.cnsns.2014.10.012

[22] A. Beléndez, M. L. Álvarez, E. Fernández, and I. Pascual, "Cubication of conservative nonlinear oscillators," European Journal of Physics, vol. 30, pp. 973-981, 2009. http://dx.doi.org/10.1088/0143-0807/30/5/006 


\section{FIGURE CAPTIONS}

Figure 1.- Exact period of the cubic-quintic oscillator as a function of the initial amplitude $A$ for $a_{1}=a_{3}=1$ and three values of $a_{5}$ : (a) 5, (b) 10, and (c) 30 .

Figure 2.- Parameter $m$ (Eq. (24)) as a function of the initial amplitude $A$ for $a_{1}=a_{3}=1$ and three values of $a_{5}$ : (a) 5, (b) 10, and (c) 30 .

Figure 3.- Variation of the nonlinear function $f$ (or parameter $q_{1}$ ) as a function of the initial amplitude $A$ for (a) $a_{1}=1, a_{3}=-\sqrt{40}$ and $a_{5}=10$ and for (b) the cubic-quintic oscillator $a_{1}=1, a_{3}=0$ and $a_{5}=10$.

Figure 4.- Period as a function of $A$ for $a_{1}=1, a_{5}=10$ and five values of $a_{3}$ : (a) 0 , (b) -3 , (c) -5, (d) -5 , and (e) $-\sqrt{40}$.

Figure 5.- Period for $a_{1}=1$ and $a_{5}=10$, as a function of $A$ and $a_{3}$, where it can be seen that the exact equations for the period are applicable for all values of $A$ only if $a_{3}>-\sqrt{40}$.

Figure 6.- Period for $a_{1}=1, a_{3}=-\sqrt{40}$ and $a_{5}=10$, as a function of the initial amplitude A.

Figure 7.- Parameter $m$ for $a_{1}=1, a_{3}=-\sqrt{40}$ and $a_{5}=10$, as a function of the initial amplitude $A$.

Figure 8.- Period for $a_{1}=1, a_{3}=-6.324$ and $a_{5}=10$, as a function of the initial amplitude $A$. The period reaches a maximum value of 88.73 for $A=0.564$

Figure 9.- Parameter $m$ for $a_{1}=1, a_{3}=-6.324$ and $a_{5}=10$, as a function of the initial amplitude $A$.

Figure 10.- Period for $a_{1}=1$ and $a_{5}=10$, as a function of $A$ and $a_{3}$, with $a_{3} \geq-6.324$.

Figure 11.- Exact periodic solutions for the cubic-quintic Duffing oscillator for $a_{1}=1, a_{3}=$ -6.324 and $a_{5}=10$, and different values for the initial amplitude: (a) $A=0.1$, (b) $A=0.5$, (c) $A=0.564$, (d) $A=0.65$, (e) $A=0.725$, and (f) $A=1$. 
Figure 12.- Exact velocity of the cubic-quintic Duffing oscillator for $a_{1}=1, a_{3}=-6.324$ and $a_{5}=10$, and different values for the initial amplitude: (a) $A=0.1$, (b) $A=0.5$, (c) $A=0.564$, (d) $A=0.65$, (e) $A=0.725$, and (f) $A=1$.

Figure 13.- Phase plot for $a_{1}=1, a_{3}=-6.324$ and $a_{5}=10$ and for (a) $A=0.1$, (b) $A=0.5$, (c) $A=0.75$, and (d) $A=1$.

Figure 14.- First four Fourier coefficients as a function of $A$ for $a_{1}=1, a_{3}=-6.324$ and $a_{5}=$ 10. (a) $c_{1}$, (b) $c_{3}$, (c) $c_{5}$, and (d) $c_{7}$ 


\section{TABLES}

Table 1. Values of the first four coefficients of the Fourier series expansion of the exact solution for values of $A$ corresponding to in Figure 11.

\begin{tabular}{|r|r|r|r|r|r|r|}
\hline & (a) $A=0.1$ & (b) $A=0.5$ & (c) $A=0.564$ & (d) $A=0.65$ & (e) $A=0.725$ & (f) $A=1$ \\
\hline$c_{1}$ & 1.00203 & 1.11964 & 1.25004 & 1.05331 & 0.961324 & 0.904146 \\
\hline$c_{3}$ & -0.00203238 & -0.143846 & -0.373948 & -0.106919 & -0.000747937 & 0.073435 \\
\hline$c_{5}$ & $6.85722 \times 10^{-6}$ & 0.029753 & 0.197055 & 0.0589628 & 0.0342675 & 0.0179816 \\
\hline$c_{7}$ & $-2.50124 \times 10^{-8}$ & -0.006890971 & -0.120240 & -0.0100617 & 0.00277384 & 0.00347569 \\
\hline
\end{tabular}


FIGURE 1

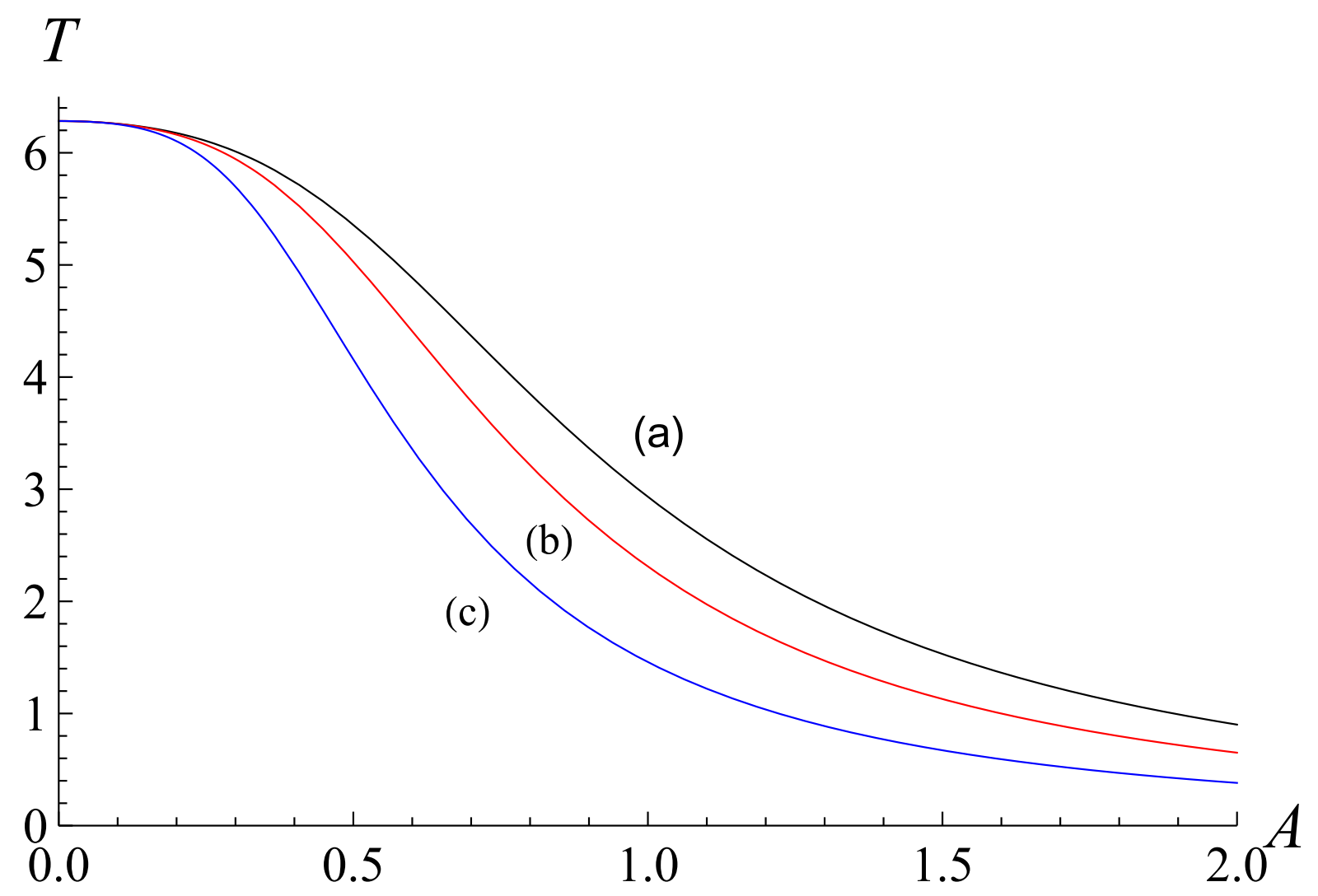


FIGURE 2

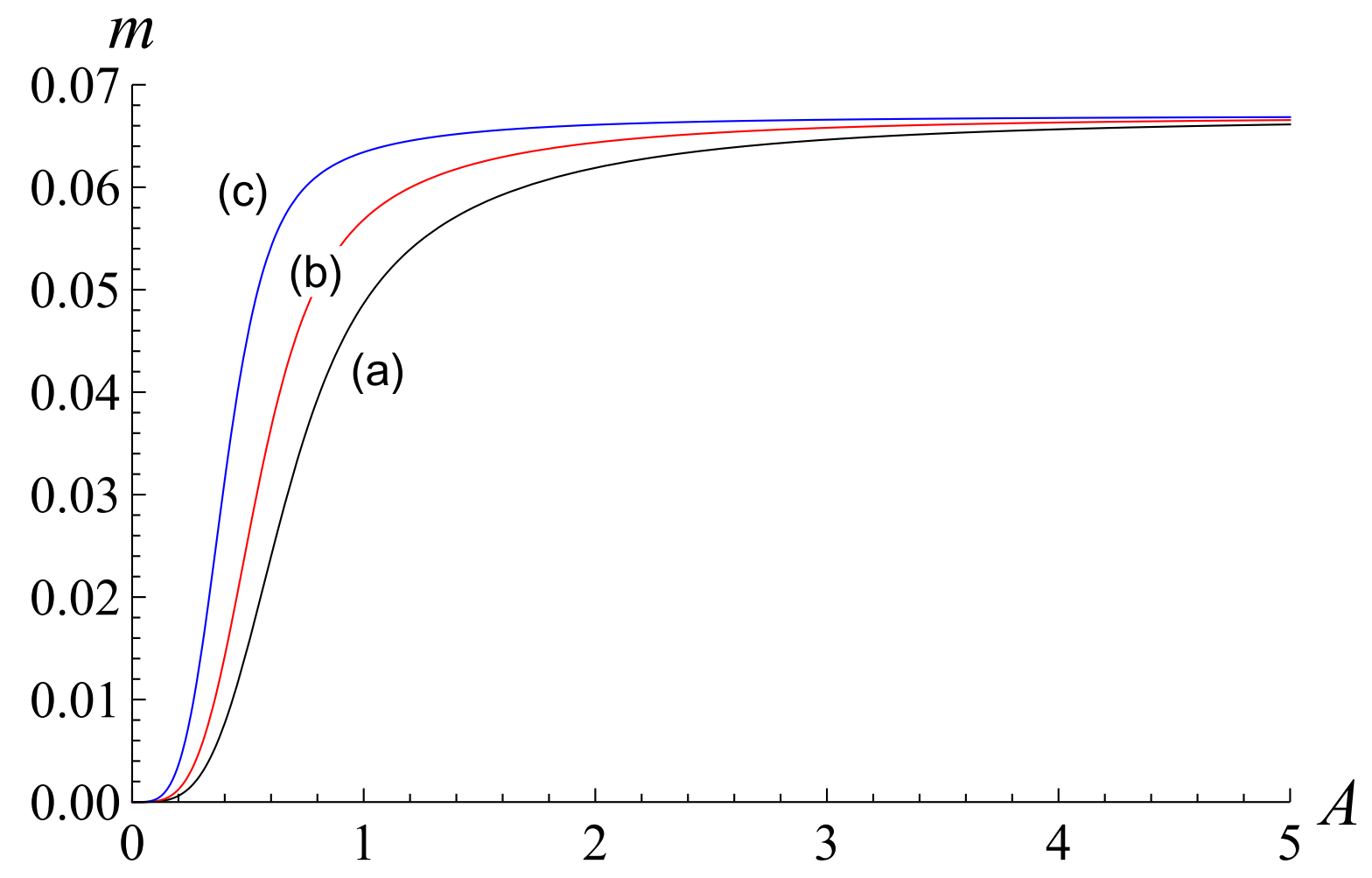


FIGURE 3

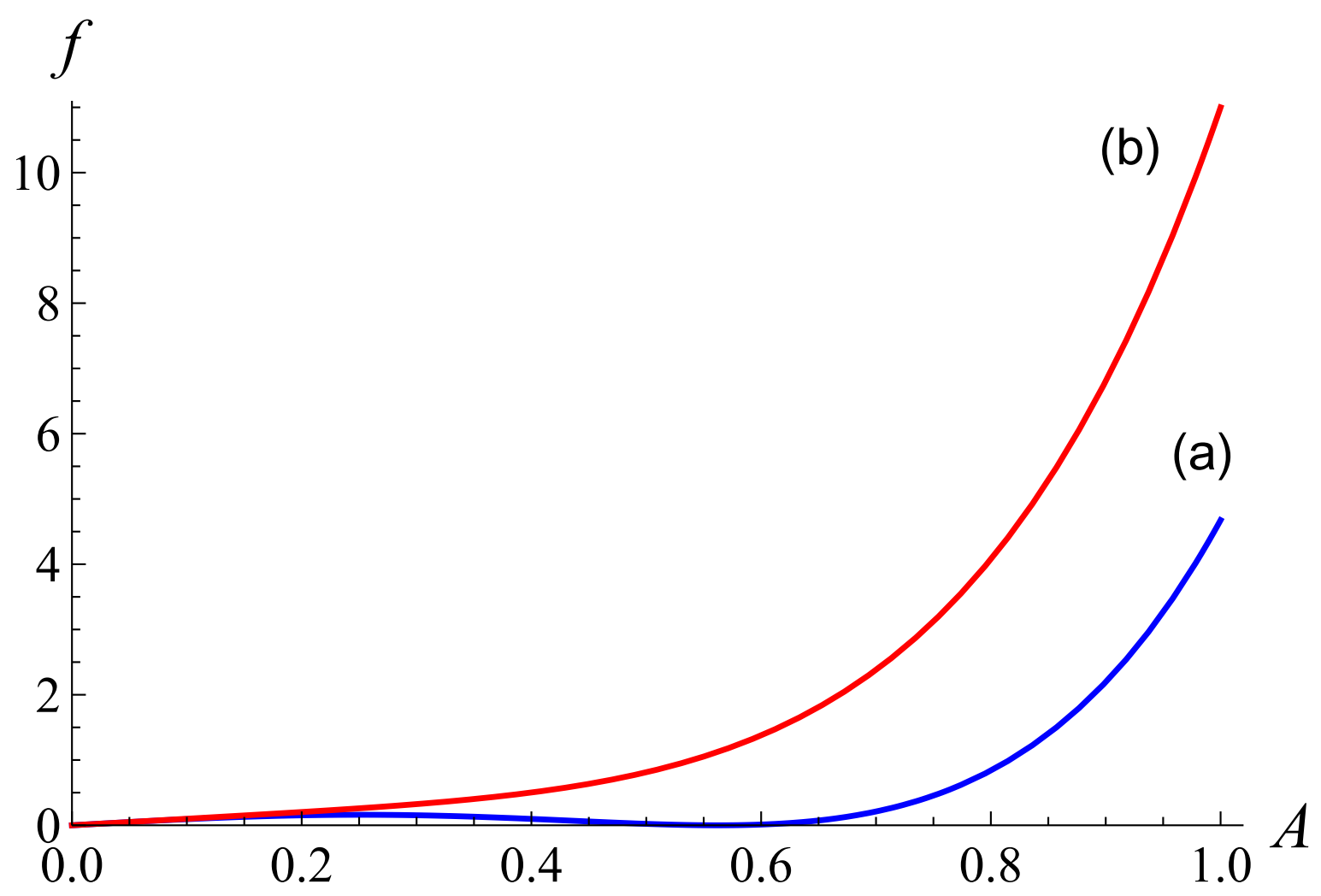


FIGURE 4

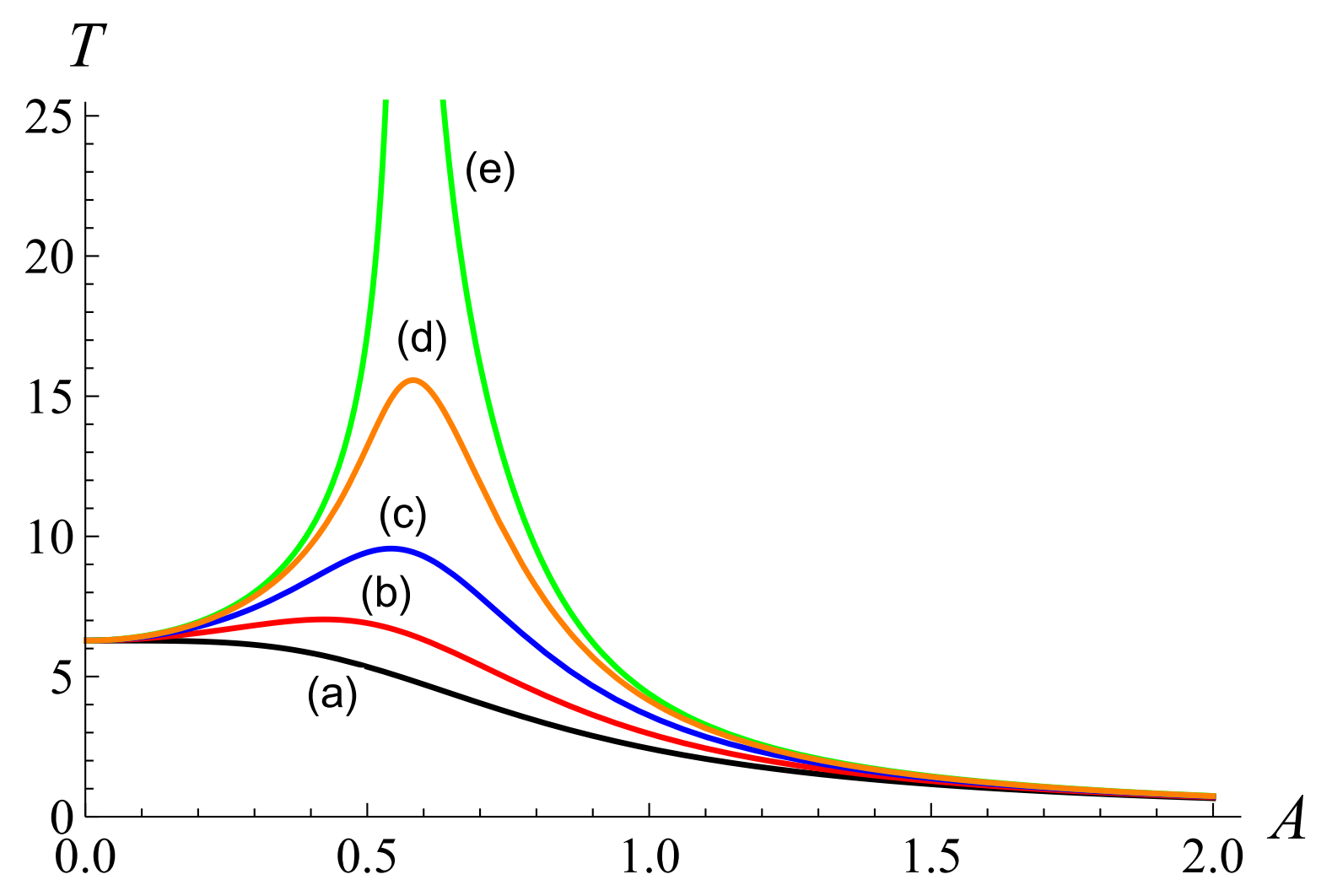




\section{FIGURE 5}

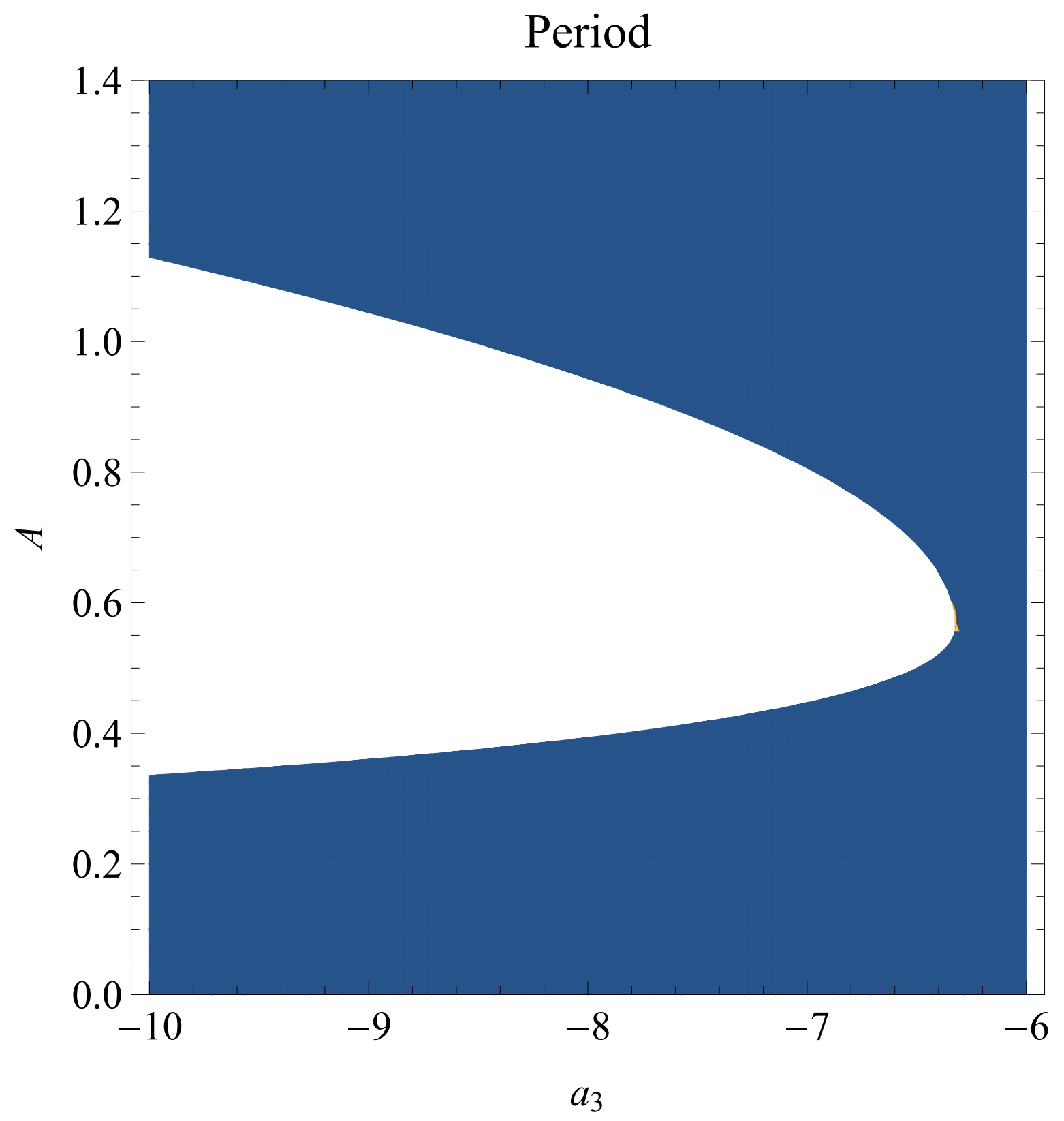


FIGURE 6

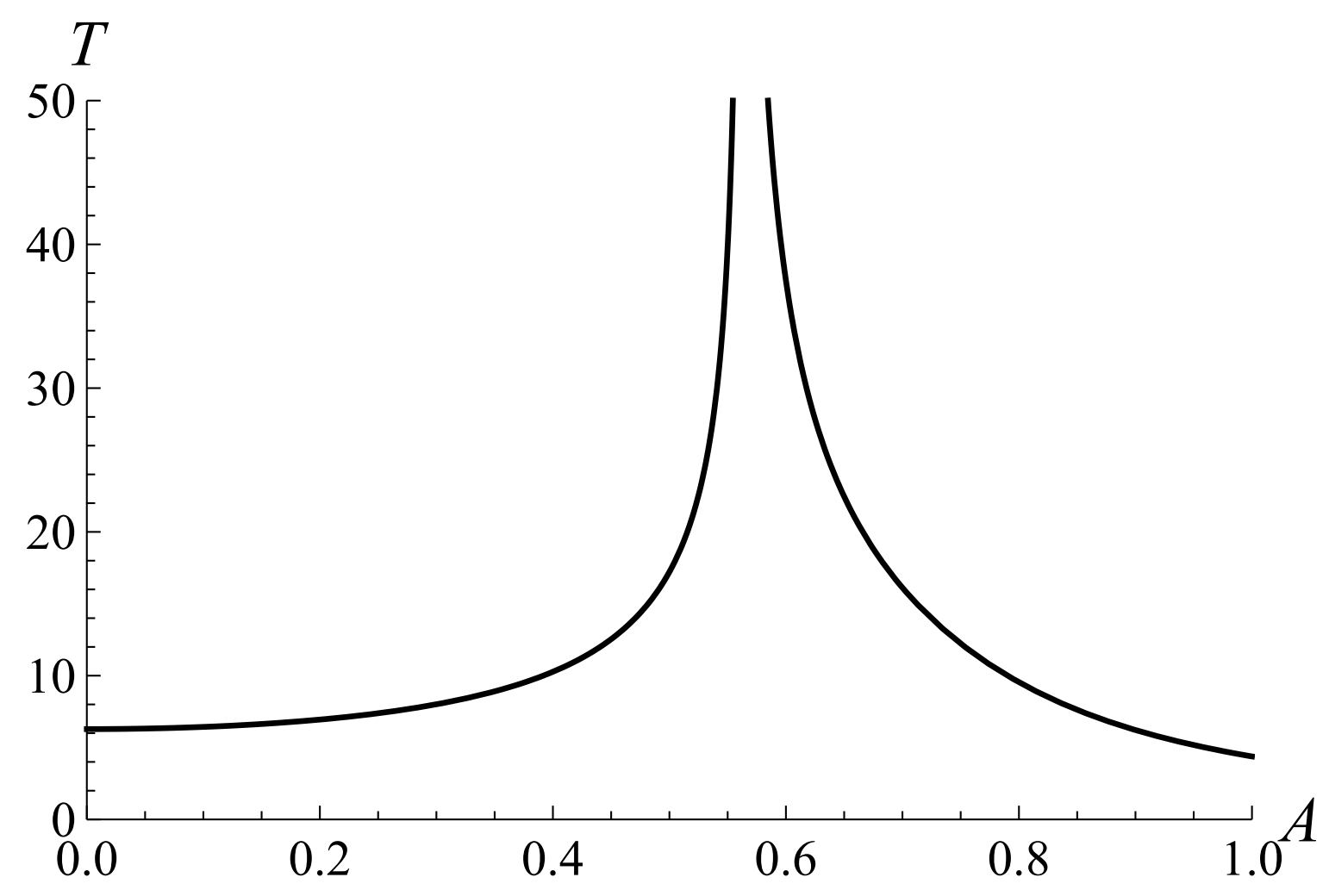


FIGURE 7

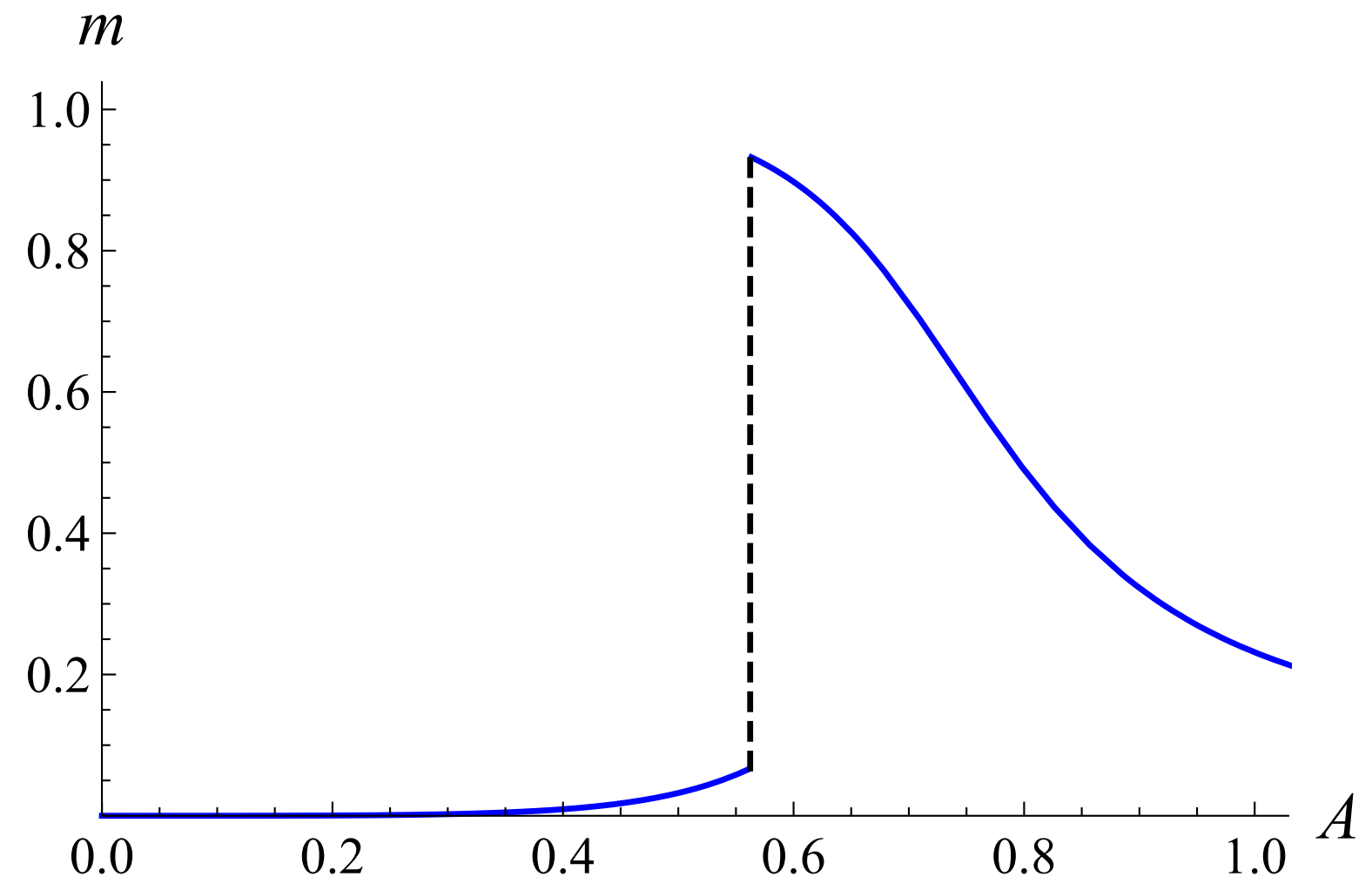


FIGURE 8

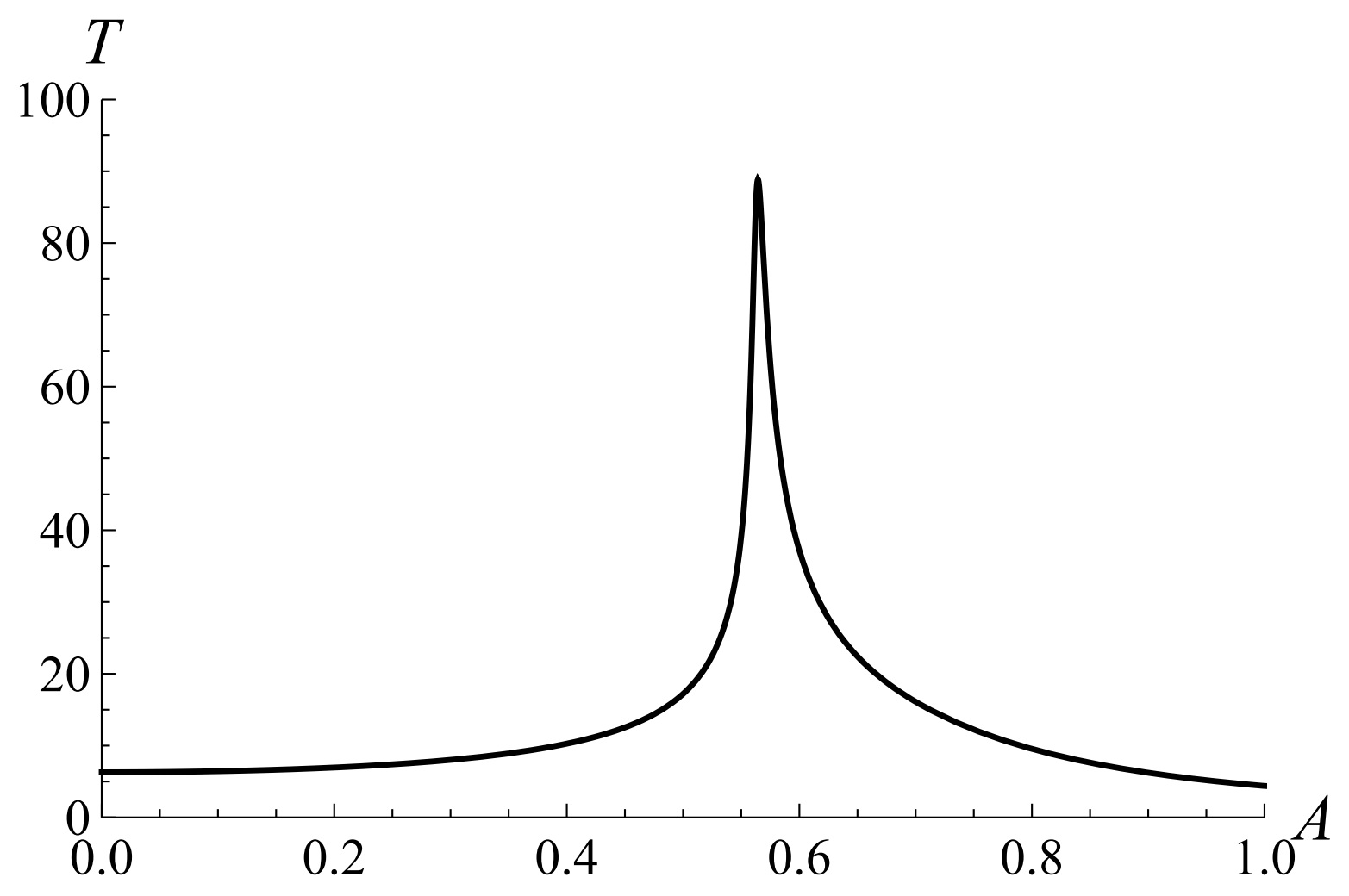


FIGURE 9

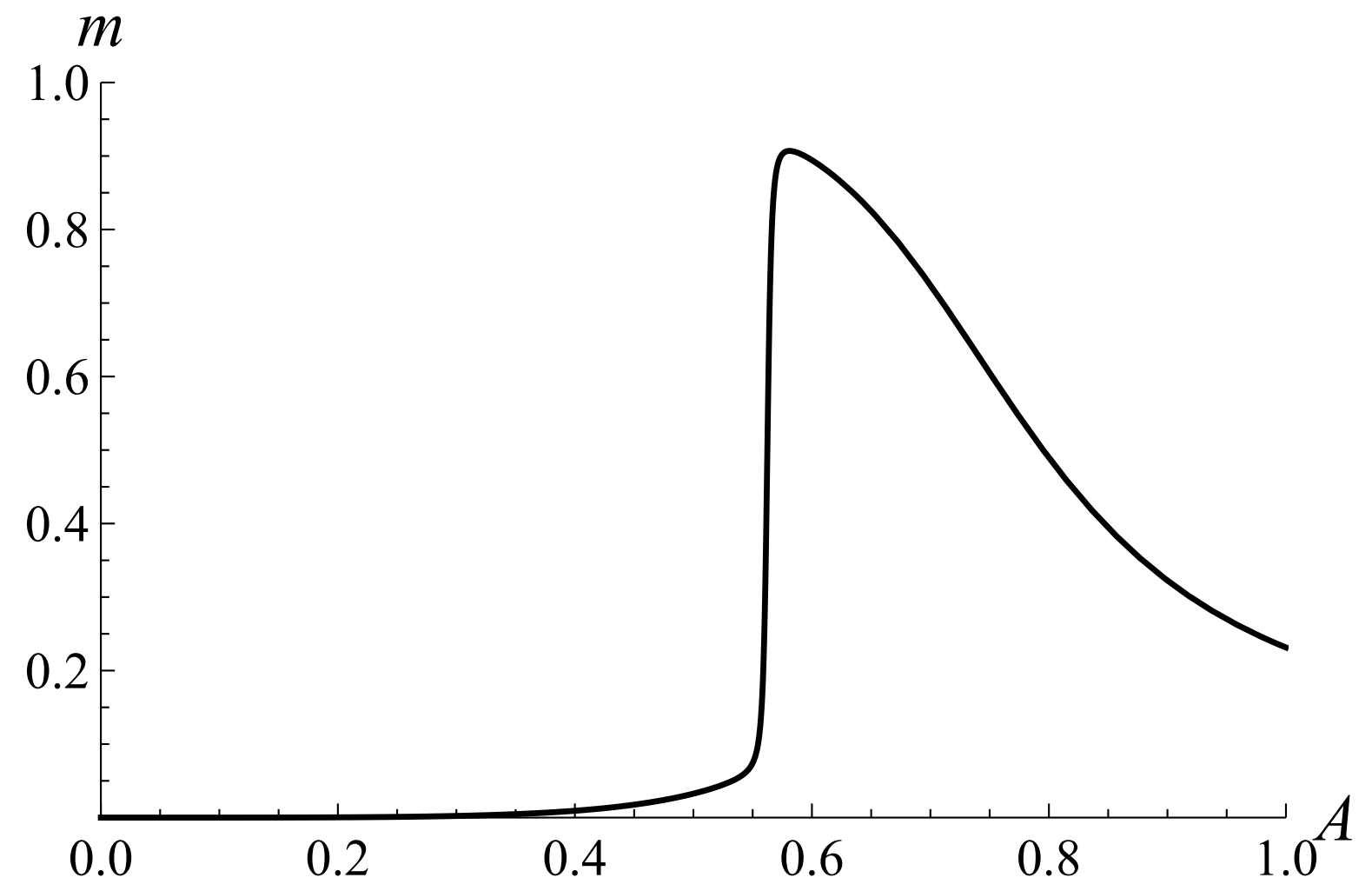


FIGURE 10

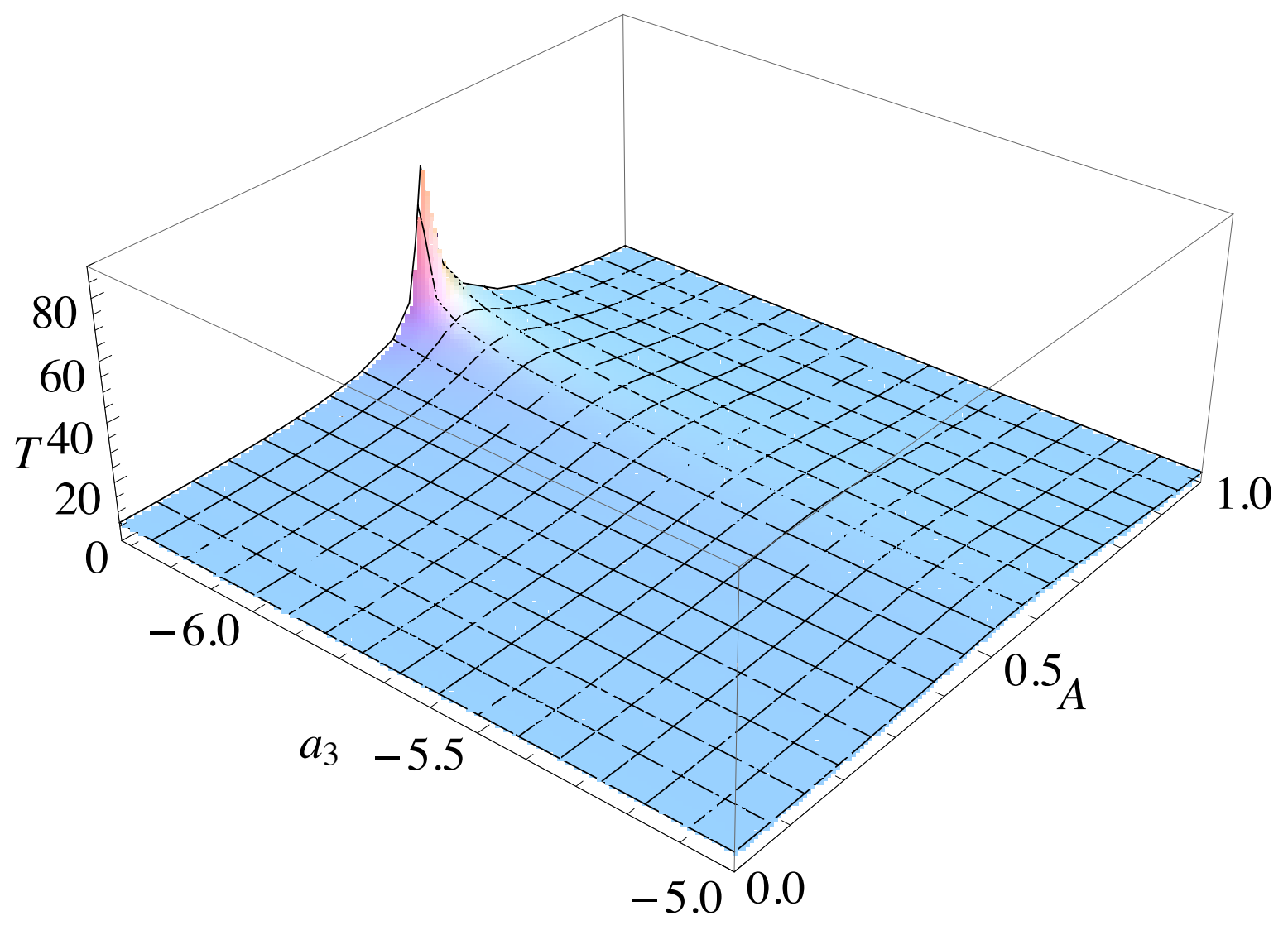


FIGURE 11

(a)

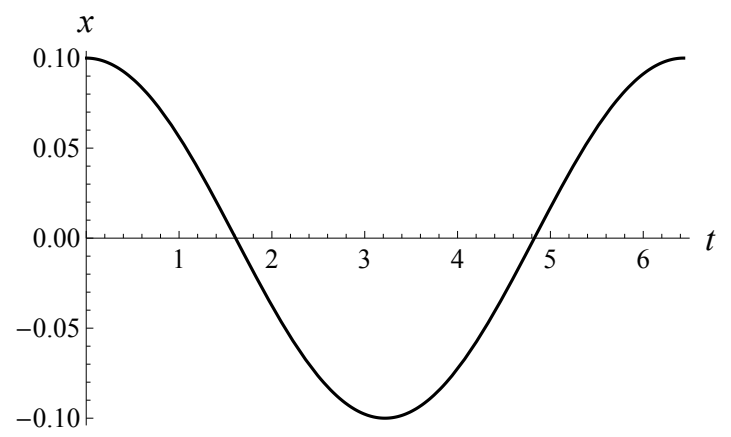

(c)

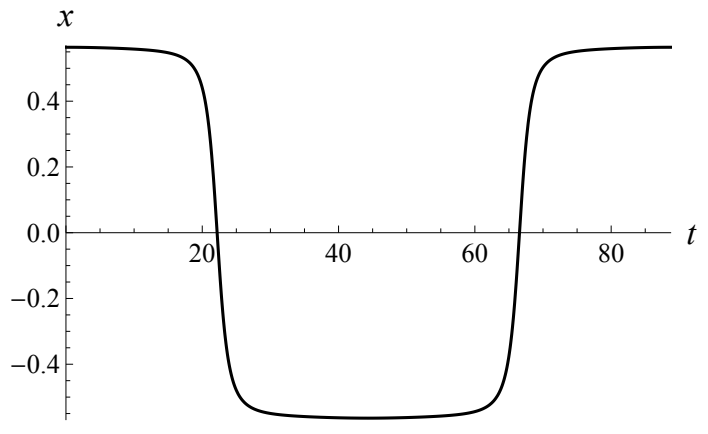

(e)

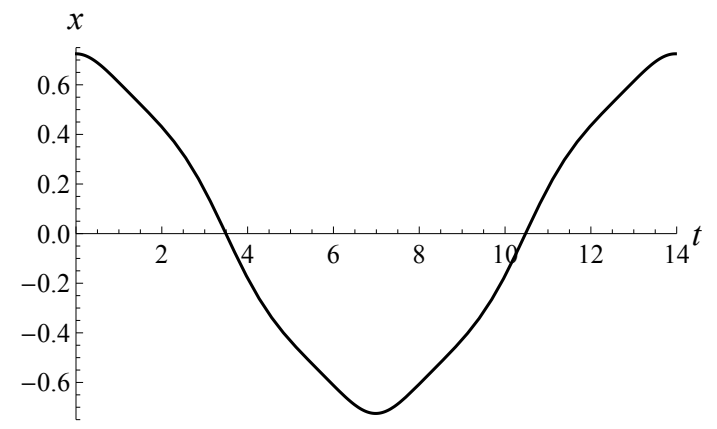

(b)

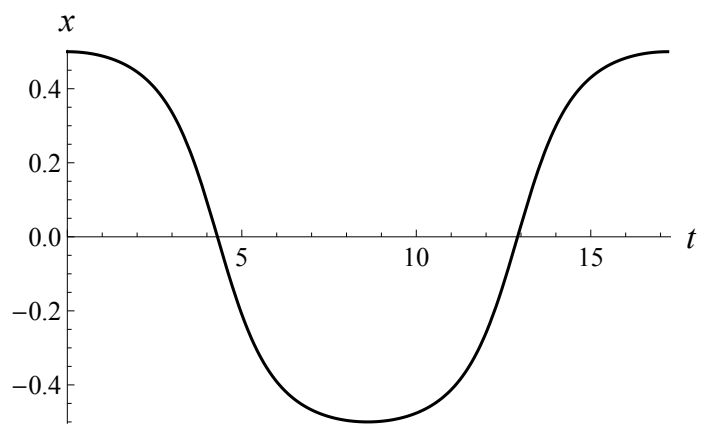

(d)

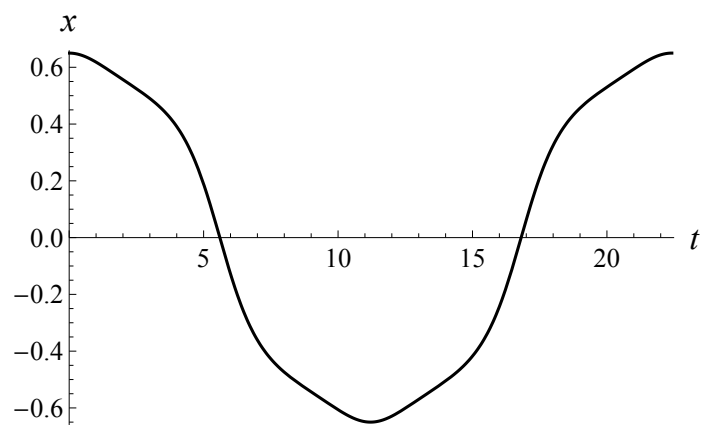

(f)

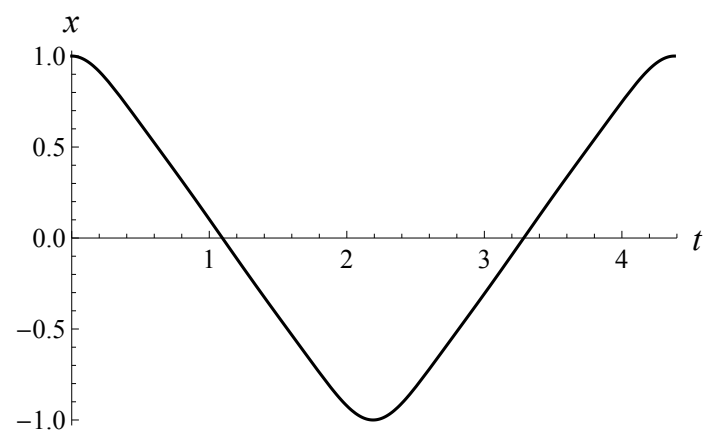


FIGURE 12

(a)

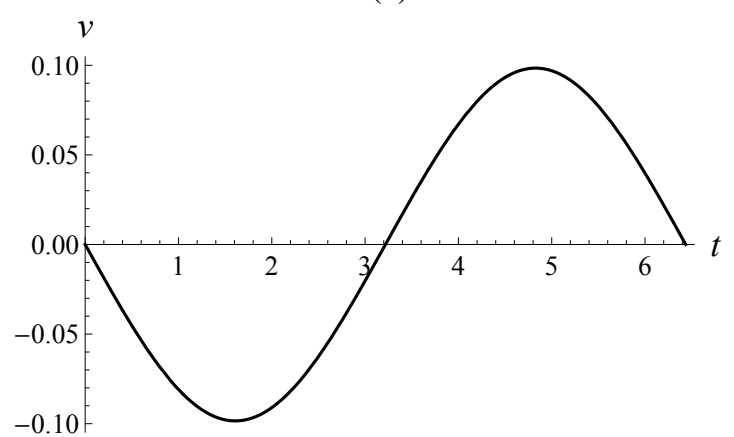

(c)

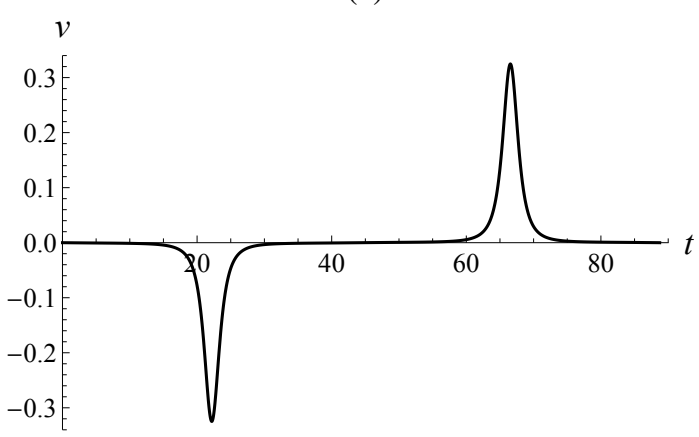

(e)

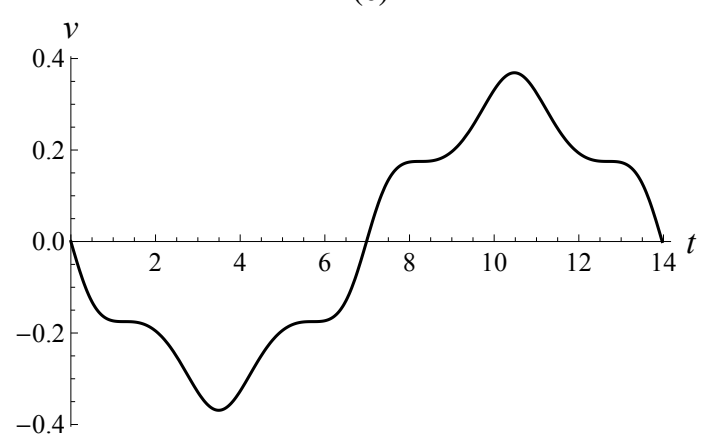

(b)

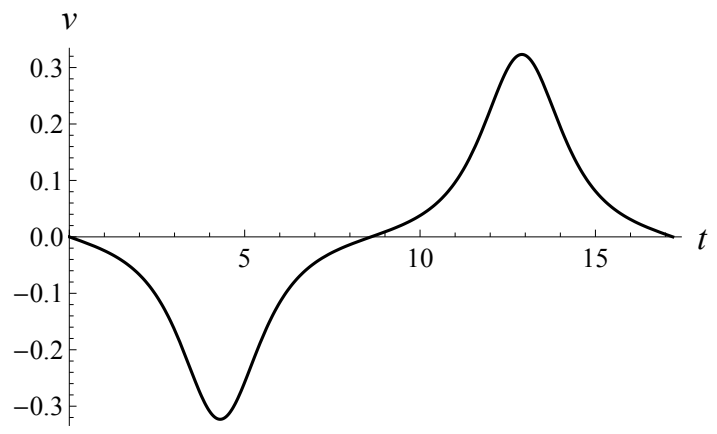

(d)

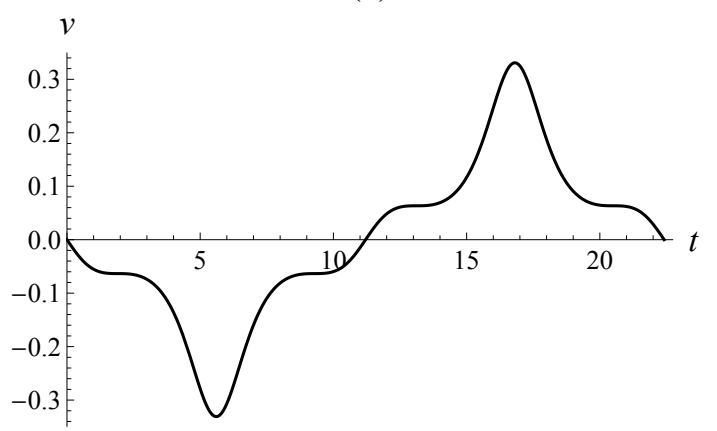

(f)

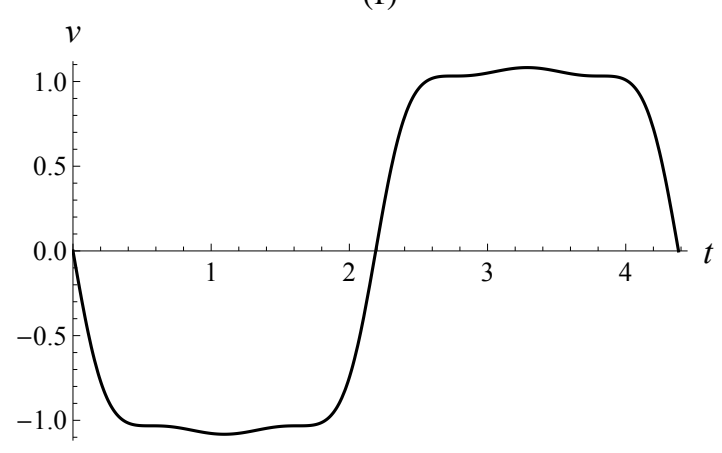


FIGURE 13

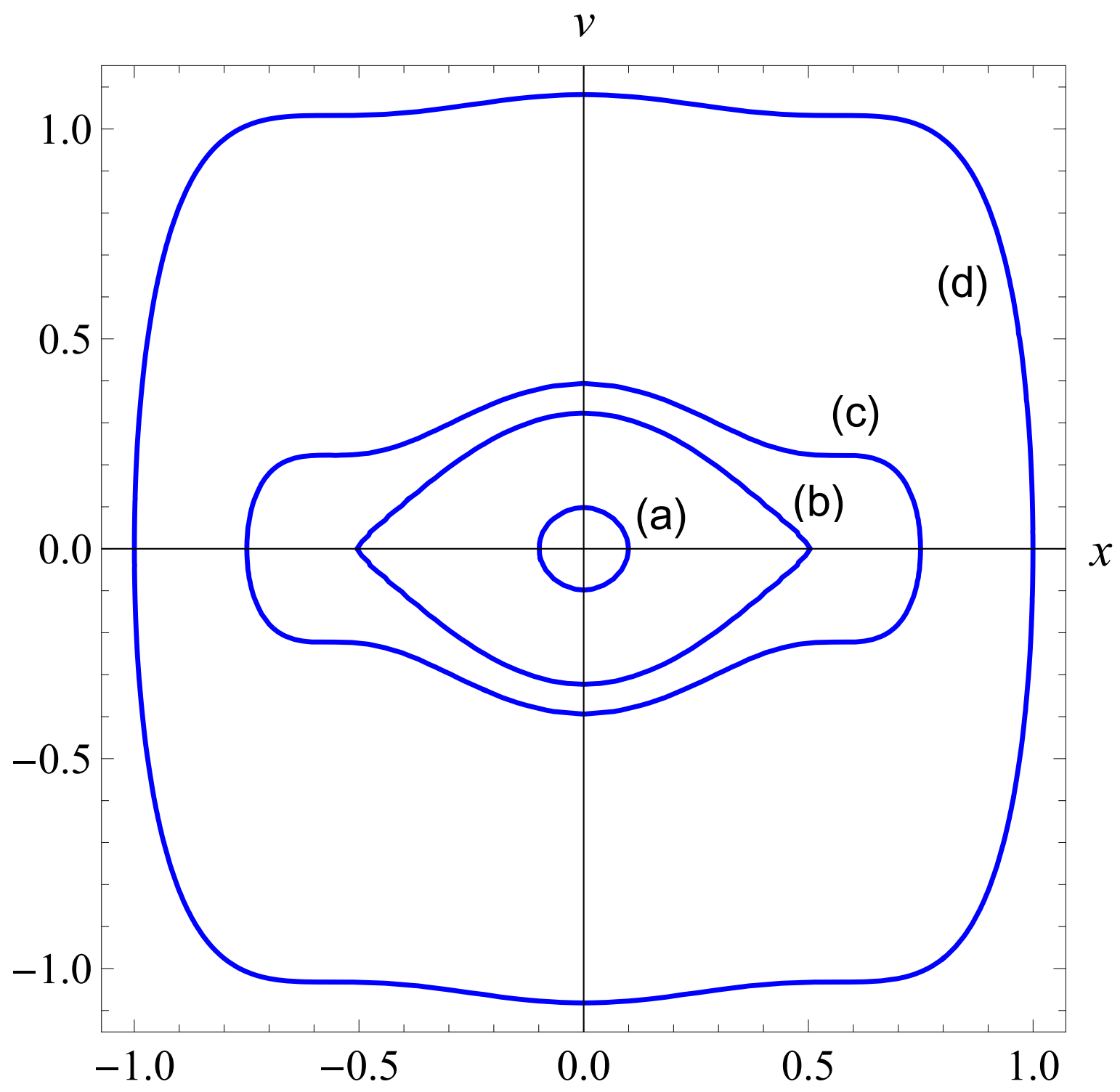


FIGURE 14

(a)

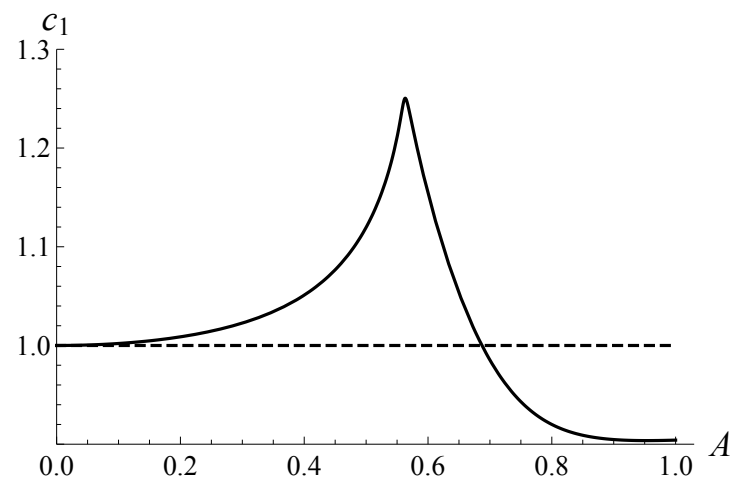

(c)

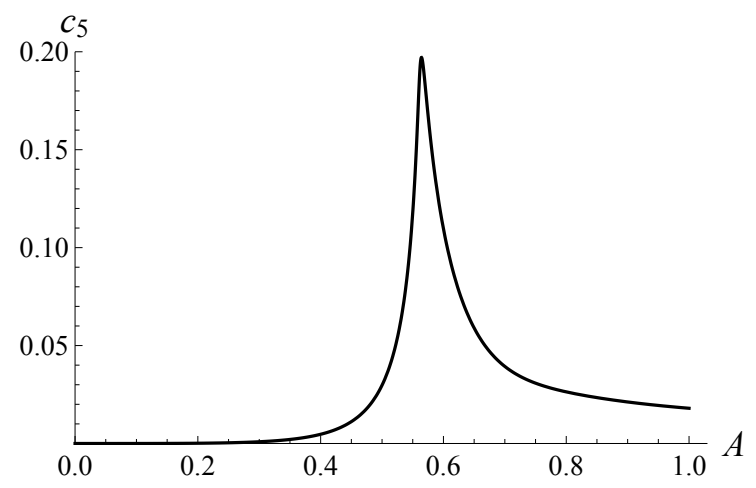

(b)

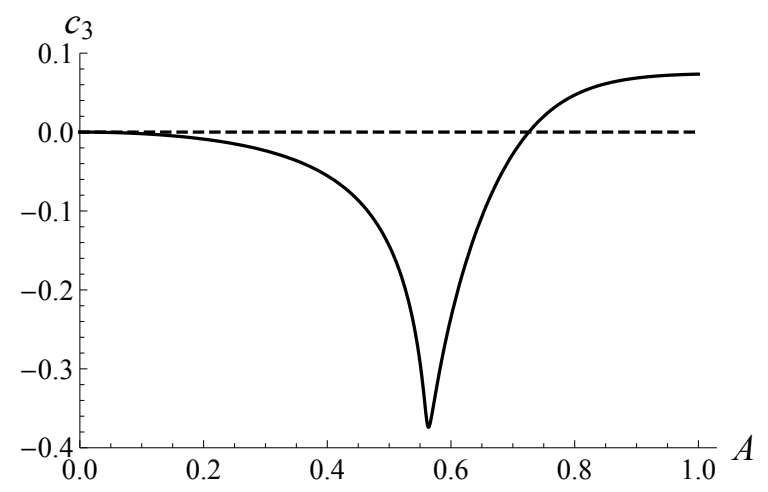

(d)

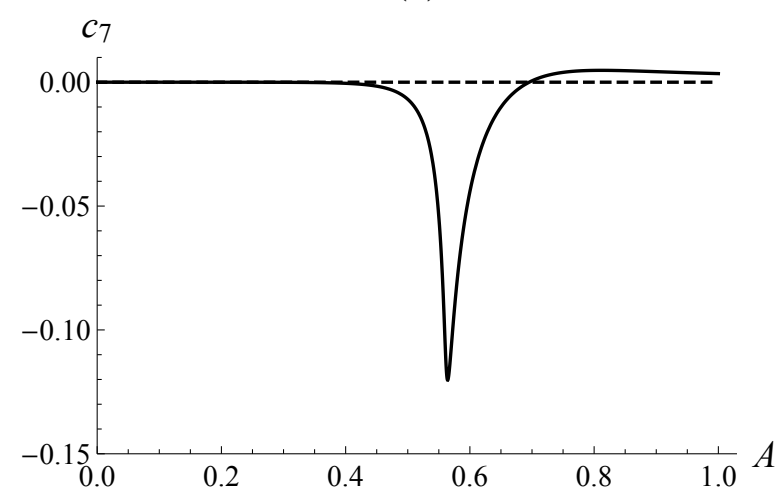

